\title{
A review on the recent energy-efficient approaches for the Internet protocol stack
}

\author{
Korhan Cengiz ${ }^{1,2}$ and Tamer Dag ${ }^{3 *}$
}

\begin{abstract}
The reduction of energy consumption has become a key research area for the information and communication technology (ICT) industry, due to economical, environmental, and marketing reasons. While the environmental direction aims at minimization of greenhouse gas emissions by enforcing the usage of renewable energy in the ICT industry, economical and marketing directions lead researchers to design low-power components or develop and enhance energy-saving protocols without an impact on the level of the performance. With the steady increase in the cost of energy, the expanding number of energy-hungry components and widespread usage of ICT industry, most of the protocols that have become an integral part of our lives but are yet developed without any energy constraints in mind in the past will need to be restructured or developed again. For this reason, researchers are studying on all layers of the Internet protocol stack to develop energy-efficient protocols and algorithms. This paper reviews recent approaches for energy efficiency studies for each layer in the Internet protocol stack from the physical layer to the application layer. It is expected that with the deployment of current research output, the studies performed at each layer will result in significant energy savings for the ICT industry which in turn will have a positive impact on our lives for their economical and environmental results.
\end{abstract}

Keywords: Green communications; Green networking; Energy-efficient protocols; Energy-efficient applications

\section{Review \\ Introduction}

Nowadays, the information and communication technology (ICT) industry represents an important source of energy consumption and greenhouse gas emissions. Although, it is extremely difficult to configure the exact amount of energy consumption for the ICT, various resources estimate these numbers from a conservative 3\% of the worldwide electricity consumption up to two-digit figures [1,2]. Even if the above mentioned conservative figures are accepted, it is a well-known fact that the ICT industry will grow in an exploding manner and the trend for the rapid increase in the amount of energy consumption will continue. However, the excessive amount of energy consumption will not only increase greenhouse gas emissions but will also have a significant impact on global warming and climate changes.

\footnotetext{
${ }^{*}$ Correspondence: tamer.dag@khas.edu.tr

${ }^{3}$ Computer Engineering Department, Kadir Has University, Cibali, 34083 Istanbul, Turkey

Full list of author information is available at the end of the article
}

Green communication technologies are expected to reduce and/or solve the serious problems which we are very likely to embrace in the future with the emergence of new nature-friendly technologies. While the focus of the environmental view of green communications aims at minimizing greenhouse gas emissions and the focus of the engineering view of green communications tries to find ways of reducing the energy usage in the sector, both views expect similar outcomes.

Based on these facts, network and communication devices, protocols, and algorithms should be aware of the energy that they consume to make efficient and responsible decisions, as energy consumption and other green issues are fast becoming the ICT industry's biggest challenge. Many protocols that are currently in use were developed to achieve maximum performance, and the energy consumption issues were neglected. Given that the enormous growth of the ICT sector will continue, it has become extremely important to take precautions quickly and start implementing new protocols to reduce energy consumption. For this purpose, most of the existing protocols are being enhanced or new ones are being

\section{Springer}

(c) 2015 Cengiz and Dag; licensee Springer. This is an Open Access article distributed under the terms of the Creative Commons Attribution License (http://creativecommons.org/licenses/by/4.0), which permits unrestricted use, distribution, and reproduction in any medium, provided the original work is properly credited. 
developed to achieve these goals in every layer of the Internet protocol stack.

The aim of this paper is to review and summarize the recent studies for energy-efficient approaches for all the layers of the Internet protocol stack starting from the physical layer up to the application layer. Table 1 summarizes some of the work done for each of the layers. A considerable amount of research is devoted for the physical layer for energy-efficient solutions. Developing energyaware power amplifiers, modulation techniques are the focus of the physical layer studies. Most of the work completed on green communications for the data link layer is on the Media Access Control (MAC) sublayer since MAC protocols have a considerable impact on energy consumption. A MAC protocol reducing the number of collisions with effective usage of radio interfaces can result in significant savings. Another important area for conservation of energy is scheduling algorithms. Data centers are significant energy-hungry systems as their numbers, capacities, and sizes are increasing rapidly. It is estimated that data centers are responsible for approximately a quarter of the energy consumption of the overall network. Thus, many researchers have devoted their studies for minimizing energy consumption for the data centers by optimizing their locations, developing routing algorithms. Due to the bursty nature of the network traffic, a large proportion of network devices can be underutilized for some portion of their lifetimes. Thus, switching off those devices during off-peak hours or estimating the load on the devices is an important way of energy savings. Developing efficient algorithms for mobile ad hoc networks and wireless sensor networks also might help to reduce energy

Table 1 Summary of the recent energy-efficient approaches for all layers

\begin{tabular}{ll}
\hline Layers & Energy-efficient approaches \\
\hline Application layer & Energy-aware usage of web services \\
& Energy-aware mobile application designs \\
& Periodic real-time applications \\
Transport layer & Energy-efficient TCP protocols \\
Network layer & Switching-off underutilized network devices \\
& Reducing energy consumption in data centers \\
& Energy-efficient approaches for mobile ad hoc \\
& networks \\
& Energy-efficient approaches for wireless sensor \\
& networks \\
& Energy-efficient MAC protocols \\
Energy-efficient scheduling & Energy-efficient power amplifiers \\
Data link layer & Energy-efficient modulation schemes \\
\hline
\end{tabular}

consumption. The transport layer energy efficiency studies are on optimizing the TCP. In the application layer, the development of mobile applications and usage of web services and real-time applications can contribute to a decrease in energy consumption. In addition to the studies in all layers, cross-layer energy efficiency research is also gaining strength.

The remainder of this review paper is organized as follows: A discussion on the impact of the energy consumption for the ICT industry is presented in the next section. By providing various examples from different sources, it is observed that ICT energy consumption has become an important portion of the total consumption, and thus, effective measures need to be taken. The latter sections are reserved for each layer of the Internet protocol stack: physical, data link, network, and transport and application layers. For each layer, recent studies for reducing energy consumption are described. Finally, the 'Conclusions' section summarizes and concludes the paper by specifying the importance of developing energyefficient algorithms as they will have an impact not only on us but also on future generations.

\section{The impact of ICT on energy consumption}

Global warming is the increase in Earth's average surface temperature due to the effect of greenhouse gases, such as $\mathrm{CO}_{2}$ emissions. Since the early twentieth century, Earth's mean surface temperature has increased by about $1.4^{\circ} \mathrm{F}$, with about two thirds of the increase occurring in the last three decades [3]. A major portion of the global warming in the last half century has been caused by human factors as a result of emissions of greenhouse gases. Greenhouse gases can absorb and emit long wave (infrared) radiation in a planetary atmosphere. This phenomenon is known as the greenhouse effect. Energy-related $\mathrm{CO}_{2}$ emissions, resulting from petroleum and natural gas, represent a significant amount of human-made greenhouse gas emissions [4]. Worldwide $\mathrm{CO}_{2}$ emissions are expected to increase by approximately $2 \%$ annually in the next decade. Most of the increase in the emissions is expected to occur in the developing world in emerging economies [4].

According to scientific studies, if the rate of increase of greenhouse gas emission continues with the current trend, average global temperatures are projected to increase between $2^{\circ} \mathrm{F}$ and $11.5^{\circ} \mathrm{F}$ before the start of the next century [3]. The temperature increase can cause very serious and dangerous effects on the environment and human life such as accelerated sea level rise, reduction in the amount of fresh water, changes in the ecosystems, and increased amount and severity of floods and droughts, thus resulting in severe problems for all forms of life living on Earth $[5,6]$.

The ICT sector which accounts for approximately $2 \%$ of global $\mathrm{CO}_{2}$ emissions [7] has become an integral lifestyle 
commodity for human beings. ICT has played a central role in bringing the people of the globe together. People and the companies have become dependent on ICT for different kinds of reasons such as e-commerce, online banking, home shopping, and instant access to music, books, and videos. With the invention of new technologies and applications or widespread usage of emerging technologies such as Cloud Computing, Smart Cities, and Internet of Things, ICT's impact on human life will continue to increase rapidly. However, according to a report by Alcatel-Lucent [8], the network component of ICT is responsible for approximately 250 to 300 million tons of $\mathrm{CO}_{2}$ emissions which is equivalent to 50 million automobiles or $20 \%$ of the cars in the US. Thus, the ICT sector has become a major contributor to global warming, and consequently, measures need to be taken for greening the ICT. Figure 1 shows how different ICT fields contribute to global $\mathrm{CO}_{2}$ emissions.

Based on [9], ICT now uses approximately 1,500 TWh of power per year which is roughly equivalent to $10 \%$ of the world's total electricity generation or the combined power production of Germany and Japan. The power consumption of ICT is doubling every 5 years at an alarming rate. Another research by [10] illustrates the usage of phase electricity consumption for a number of ICT services as an overview of ICT energy consumption. According to this study, the collective electricity consumption of communication networks, data centers, and personal computers which are the major energy-hungry parts of the ICT is also growing rapidly. For example, in 2007, electricity consumption of these parts worldwide was less than 700 TWh. But in 2012, this amount has exceeded $900 \mathrm{TWh}$, about one-third increase only in 5 years. As the usage of ICT products and services is growing exponentially, electricity consumption will also increase in a similar manner. In 2012, phase electricity consumption of these parts exceeded $4.7 \%$ of the total electricity consumption.

In the UK, ICT equipment accounts for approximately $10 \%$ of UK's total electricity consumption according to [11]. Asami and Namiki [12] present the energy consumption for ICT equipment in Japan. The total power consumption of only the routers was 7.3 TWh in the year 2004, and in 2010, this value has reached 13 TWh. Another study [13] estimates that the electric power consumption of the 1.5 billion computers in the world is about $10 \%$ of the global consumption. Hinton et al. [14] also investigate the power consumption of ICT and illustrate the power consumption of the wired and wireless access network technologies per user as a function of the access rate and year. According to [14], wireless-based (WiMAX, 3G/UMTS) access networks are the most power-demanding technologies and the power consumption per user increases rapidly. For example, in 2010, while the power per user for WiMAX was about $8 \mathrm{~W}$, this value is tripled to $24 \mathrm{~W}$ in 2014.

Nowadays, there are more than 4 million base stations serving mobile users, and each year, 120,000 new base stations are deployed servicing 400 million new mobile subscribers around the world [15]. Each base station consumes, on the average, $25 \mathrm{MWh}$ per year. As a result, electricity has become a significant cost factor for both network operators and their users. Figure 2 shows the cellular network power consumption fields, and it can be clearly seen that the base stations and mobile switching are responsible for a significant portion of power consumption. Also, the most energy-consuming part of the

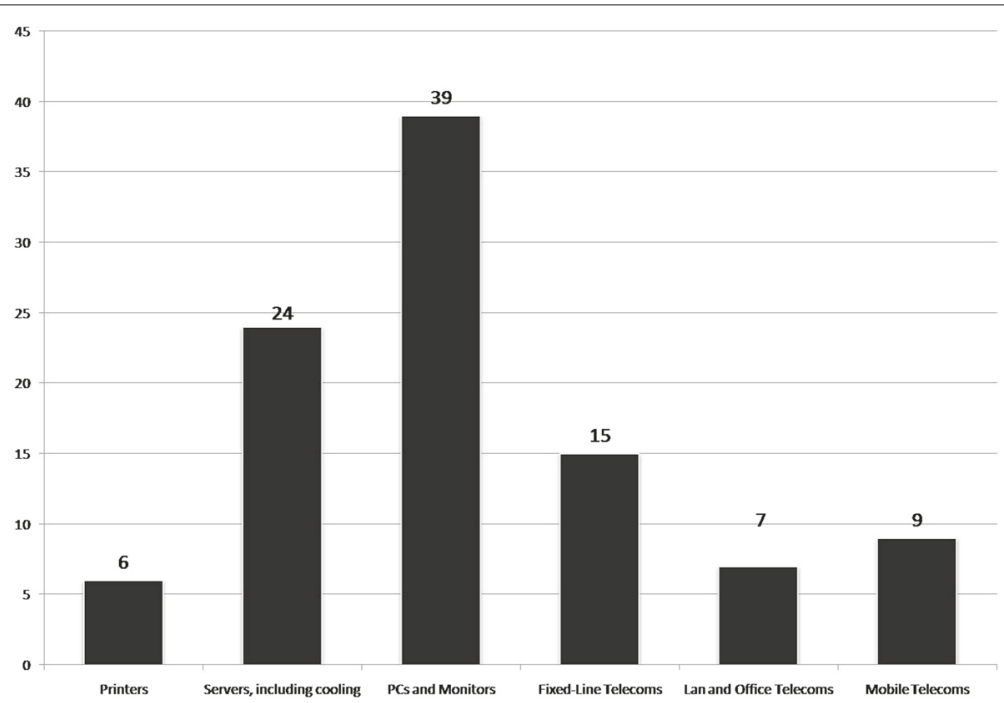

Figure 1 ICT fields' global $\mathrm{CO}_{2}$ emissions. 


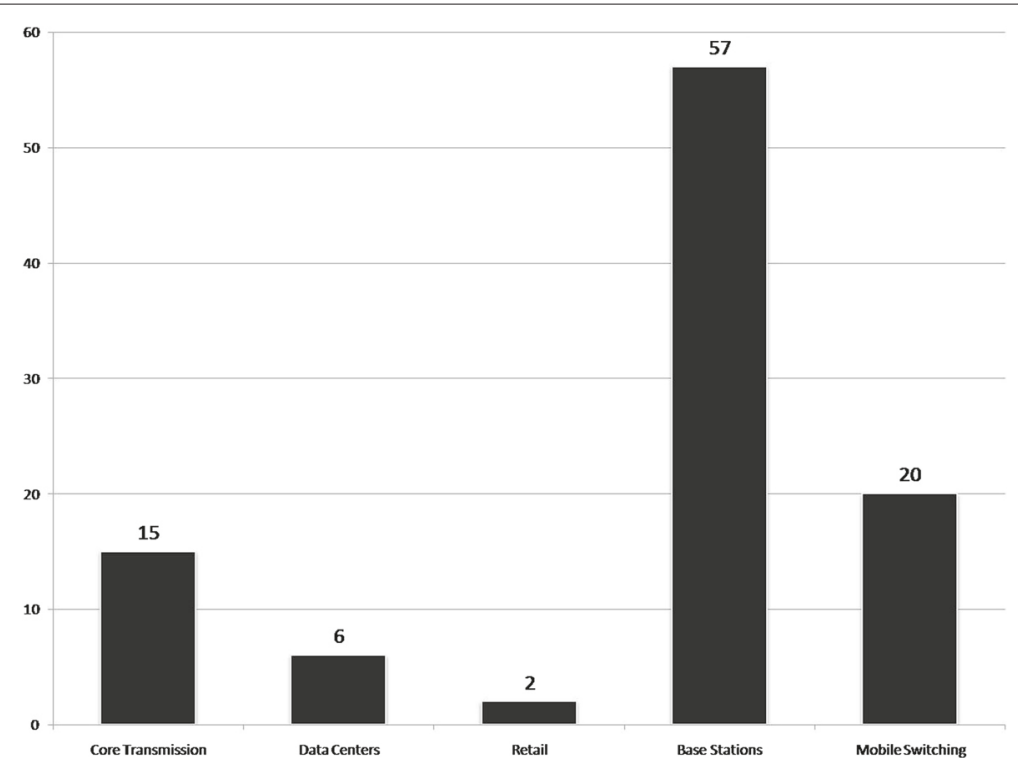

Figure 2 Cellular network power consumption.

base stations is the power amplifiers. According to the research of green wireless networks [16], power amplifiers cause more than half of the energy consumption of the base stations.

The Internet traffic is also rapidly increasing. Currently, the total annual traffic is at a level measured in exabytes ( $10^{18}$ bytes), and it is expected to reach zettabyte $\left(10^{21}\right.$ bytes) levels in the next 5 years. The energy consumption of the Internet has continued to increase as a result of demand growth of at least 50 to 100 times in the last decade. Further demand growth between $40 \%$ and $300 \%$ is estimated in the next 3 years [17]. According to a report by $\mathrm{CNN}$ [18], more power is pumped for the maintenance of Internet than the global automotive industry. Thus, Internet uses more electrical power than the entire automotive industry's combined production of cars and trucks. If we assumed that the Internet was a country, it would rank fifth in the world for the amount of energy consumption and carbon dioxide emissions. A report by Greenpeace states that the Internet consumes more power than Russia. Greenpeace estimates that when functioning at full capacity, Google's eight server farms could use up to $476 \mathrm{MW}$ of electricity which would be enough energy to power San Diego [18] which had a population of about 1.32 million people in 2012.

The energy consumption of modern networks has reached remarkable levels in the recent years because of the increased pervasiveness of computer networks. European Telecoms nowadays consume approximately 21.4 TWh per year, and it is anticipated to increase to about 35.8 TWh by 2020 [19]. The IP networks currently are responsible for $20 \%$ of total energy consumption of the
Internet, and the prediction shows that the energy consumption of IP networks in the year 2017 will be 12 times larger than that in 2009 [20].

With the entry of software-driven smart phones, the Internet is now accessible from mobile platforms which results in even greater demand for broadband. According to [9], last year, the average smart phone customer used $1.58 \mathrm{~GB}$ of data per month. Each GB requires approximately $19 \mathrm{~kW}$. Thus, the average smart phone user consumes $361 \mathrm{kWh}$ of electricity per year.

In addition to the global energy consumption perspective of the ICT, [21] points out that a desktop computer uses approximately $200 \mathrm{kWh}$ of electricity annually, compared with 50 to $70 \mathrm{kWh}$ for a notebook PC, $12 \mathrm{kWh}$ for a tablet, or $2 \mathrm{kWh}$ for a smart phone in a residential area.

As can be seen from the above examples, it is very difficult to find out the exact energy consumption of the ICT and the estimated figures are different from each other. However, on the other hand, it is very crucial to comprehend that these figures are extremely significant and they will continue to increase rapidly. Thus, urgent actions need to be taken to reduce the severity of this problem. For all layers of the Internet protocol stack, many researchers have started working on developing new technologies, enhancing current ones for energy-efficient mechanisms. The rest of this paper will describe some of these research starting from the physical layer.

\section{Physical layer}

The physical layer consists of the basic networking hardware transmission technologies of a communication network. It is considered as the fundamental layer underlying 
the logical data structures of the higher level functions in a network and consists of radio frequency circuits, modulation, and coding. The physical layer provides an electrical, mechanical, and procedural interface to the transmission medium. A considerable amount of attention has been given to the physical layer for energy-efficient solutions.

Modulation is the process of varying one or more properties such as amplitude, phase, or frequency of a periodic signal which usually contains information. With modulation, a signal can be carried inside another signal that can be physically transmitted. Many different analog and digital modulation techniques constitute the basis for communication; thus, a considerable amount of effort is being spent for energy-efficient modulation techniques.

Power amplifiers (PA) consume large amounts of energy and dissipate heat, and since increasing demand of larger capacity, higher data rates will increase the burden on PAs; energy efficiency issues for PAs are another major research area. In this section, we discuss some of the recent energy-efficient approaches in the physical layer.

PAs have low energy efficiency because of the linearity requirement enforced by the amplitude phase modulation schemes. Introducing nonlinearity might be a way to overcome this problem; however, its consequence is distortion, and some studies for distortion cancellation [22] aim to enhance the energy efficiency of PAs by taking into consideration the polarization state of signals that is robust to nonlinear distortion and propose a polarization modulation design in wireless communications. In performance analyses, the energy efficiency of the proposed scheme is compared with phase shift keying (PSK) modulation and significant reduction in energy consumption is observed. Wei et al. [23] aim for an energy-efficient subcarrierpower allocation (EESA) scheme. EESA is implemented for the polarization-amplitude-phase modulation (PAPM) in the channel with polarization mode dispersion (PMD). In this scheme, the PA energy efficiency is optimized by distributing the PA input power on each subcarrier. According to the results, the energy efficiency of the PA is increased and significant energy gain is achieved.

Multiple-input multiple-output (MIMO) systems provide capacity and spectral efficiency improvements compared to single-antenna systems. Multiple RF chains cause an increase in energy consumption at the transceivers. Thus, decreasing the number of RF chains increases energy savings. The transmit antenna selection method is the fundamental method for single RF chains. In recent years, the spatial modulation procedure is used for utilizing multiple antennas with only one RF chain. In [24], a feedback-based spatial modulation technique is introduced by using only one RF chain. The proposed scheme results in energy savings compared to the single RF transmit antenna selection scheme.
In [25], adaptive modulation and dynamic signal-tonoise ratio (SNR) adjustment is combined for achieving an energy-efficient orthogonal frequency division multiplexing passive optical network (OFDM-PON). According to the results, a significant amount of energy efficiency is obtained for each bit in an FPGA-based receiver. In [26], linearly modulated signals are transmitted with peak power limitation. Several modulation formats are evaluated in terms of mutual information and compared from the energy efficiency perspective.

An ultra-compact, slight power modulation technique in a wireless transceiver by controlling transmit power for enhancing network power gains is designed in [27]. The on-off keying (OOK) modulator which provides low power dissipation underlies the proposed scheme, and significant energy savings are achieved.

An energy-efficient modulation consideration for wireless sensor communications is presented in [28]. In this study, an energy-efficient modulation scheme, AQDBPSK, is investigated. Its performance is evaluated in AWGN and Rayleigh fading conditions. It provides the best bit error rate (BER) performance; thus, the proposed scheme may be a good option for energy-efficient communication systems.

In [29], the cooperative diversity at physical layer is analyzed by using an energy-efficient model for the reachback problem. In this problem, a large number of sensors are scattered on a field for measuring the state of some physical process that unfolds over the field and then cooperatively send this information back to a distant receiver for further processing. In this study, the BER of the destination is analyzed and the total energy consumption for each bit is obtained. The total energy absorption for achieving a given BER with ideal delay is obtained through numerical analysis under different simulation parameters. According to the simulation results, the total energy consumption of cooperative diversity with two nodes is more than that of the direct transmission for short transmission distances, because of the circuit energy consumption for short distance transmission. However, when the distance is long enough, cooperative diversity can save more energy than direct transmission.

Energy efficiency in wireless ad hoc networks with two realistic physical layer methods is discussed in [30]. These methods are the total energy consumption calculation and the reciprocal of the energy efficiency function. The authors investigate these methods in the Rayleigh fading and lognormal fading scenarios to derive the optimum transmission range in wireless ad hoc networks. In the two-node model, the hop-by-hop retransmissions (HHRs) model is used. In the HHRs model, a packet is retransmitted between two nodes until it is received and acknowledged correctly. The transmission ranges of the nodes are adjustable. Therefore, each node has different 
levels of transmission power, and the transmission power arrangement provides energy efficiency. An energy efficiency function which minimizes the energy consumption between the source and the destination nodes is also introduced. When this function is maximized, the optimal energy consumption between the source and the destination is obtained. The reciprocal of this function is linearly proportional to the total energy consumption value. According to simulation results, the two methods - the total energy calculation method for the end-to-end model and reciprocal of the energy efficiency function method - have the equivalent energy efficiencies.

In [31], an optimization scheme based on adaptive modulation and power control for a green routing protocol is used. In order to find the energy consumption of the data packet transmission, the energy consumption per bit for transmitting these packets is calculated. The derived equation demonstrates that the transmit power depends on the distance between the transmitter and the receiver. M-ary Quadrature Amplitude Modulation (M-QAM) provides better spectral efficiency, and the $M$-ary Frequency Shift Keying (M-FSK) warrants power efficiency. Using M-FSK is the most optimum solution for sensor networks because it requires the least circuit complexity, and for a given symbol error rate (SER), it needs less transmitted power. Based on this, the non-coherent M-FSK (NC M-FSK) is selected for modulation. Total energy consumption is calculated based on two energy spending reasons. The first reason is due to the RF signal generation and target distance. The second one is due to the hardware electronic components. In the network model, each node is stationary and knows its location by using GPS. The IEEE 802.15.4 standard is taken as a reference, and the ISM band is assumed for operating the modulation schemes. For evaluating the performance of the proposed algorithm over the multi-hop route, two scenarios are chosen: without optimization of power and modulation order and with optimization of power and modulation order. In the simulations, the impact of route length is analyzed. According to results, using maximum power while transmitting at a fixed rate causes the largest energy consumption. With optimization, the system achieves significant energy gains.

In [32], a method for a robust and energy-efficient physical layer for wireless sensor networks is proposed. For assessing the radio link quality and calculating the energy per bit transmitted successfully, the BER is used. The results are presented for an indoor environment. For energy efficiency, transmission power evolution is tested for two transmission chains (with and without forward error correction), three modulation schemes (ASK, FSK, MSK), and two different frequency bands (433 and $868 \mathrm{MHz}$ ). The best configuration observed for optimal energy efficiency is a transmission system with
MSK modulation, $433 \mathrm{MHz}$ frequency band, and forward error correction (FEC) usage. It is also pointed out that the physical layer optimization alone is not suitable to achieve the desired lifetime for a wireless sensor network.

The authors of [33] had earlier introduced a novel recursive physical layer security scheme. In [33], a novel synchronization process is introduced where energy spending is minimized and the impact on memory and CPU is measured. The analysis which is confirmed by simulations sustains significant performance enhancements. The best physical layer authentication preamble (AP) consisting of a hash function using some secure keys and additional information is achieved. Through simulations, it is shown that the developed protocol is able to measure the optimum operational point where the energy dissipation of the system is minimum. The simulation results show that their system saves a significant amount of energy.

Some energy efficiency studies on the physical layer $[34,35]$ also try to combine energy efficiency with security considerations. Zhang et al. [34] combine the physical layer secrecy and application layer secrecy requirements for characterizing the partial secrecy metric to provide an energy-efficient physical layer security in Gaussian fading channels. In [35], for compensating the relaying of the information in a secret and efficient scheme and taking into consideration the quality of service (QoS) limitations, the physical layer security technique with an energy-efficient power allocation is proposed.

Packet loss, delay, and bandwidth are the parameters which can affect the performance of VoIP services. Increasing the transmission power overcomes this problem, in the expense of more energy consumption. In [36], an energy-efficient transmission power algorithm (TPO) which improves the energy utilization by considering QoS limitations is proposed. The proposed algorithm adopts the lowest possible transmission power with the help of SNR approximation without affecting the VoIP service quality. The transmission power is managed in accordance with channel conditions. In simulations, significant energy gains are obtained especially in a noisy channel situation.

\section{Data link layer}

Most of the studies for green communications for the data link layer focus on the MAC sublayer. In this section, we discuss recent energy-efficient MAC protocols as well as Long-Term Evolution (LTE) solutions.

\section{Media access control}

MAC data communication protocol is considered as a sublayer of the data link layer. A MAC protocol defines the rules of how a frame is transmitted on a communication link by addressing and channel access control 
mechanisms. When multiple nodes share a single link, the MAC protocol coordinates the channel access. This channel may provide unicast, multicast, or broadcast communication services. In this section, recent energy-efficient approaches for the MAC sublayer are discussed.

For mobile devices, the most energy-hungry component is the radio interface. For example, the radio interface approximately consumes $70 \%$ of the total energy in a smart phone when the screen is off. The radio interface can be in transmit, receive, idle, or sleep mode, and the most power is used in the transmit mode. In the idle mode, a node requires to sense the medium and therefore the consumed amount of power is approximately equal to the receive mode. Collisions are another reason of high energy consumption in wireless devices. The reduction of collisions can enhance the throughput and decrease the energy consumption. As MAC protocols assign a channel to the nodes in the network, they directly control the radio interface operation and have a significant role in energy consumption for wireless devices.

In [37], a novel energy-efficient MAC scheme is studied. The proposed scheme schedules the active and sleep times of node radio interfaces in a distributed method. The collisions are reduced by the use of a temporary coordinator node. The network throughput is improved, and a lower packet delay than current power saving mechanisms is obtained. In performance analyses, the proposed scheme is compared with a power saving mechanism in IEEE 802.11 which is called as PSM where time is divided into beacon intervals that are used to synchronize the nodes and all the nodes must stay awake for a fixed time, and IEEE 802.11 without power saving mechanism (WoPSM). Aggregate throughput, power consumption, energy consumption, and packet delay are the performance measurement metrics. The power consumption analyses show that WoPSM is the most energy consumer because all the nodes are in the awake state all the time. The overall network power consumption increases as the load increases for all the schemes. It is clearly obtained that the proposed scheme outperforms both PSM and WoPSM in terms of energy efficiency and also in terms of throughput and delay.

A popular MAC standard described by IEEE 802.15.4 for wireless sensor networks and actuator networks defines two operating modes: non-beacon-enabled mode where the receivers are always awake to receive a frame and beacon-enabled mode which describes super frames where the nodes are only awake during a small part of a super frame and they sleep quite often. In the second mode, significant traffic results in frequent collisions and packet losses which causes an increase in energy consumption and low throughput. To operate IEEE 802.15.4 with low duty cycles needs MAC parameter adjustment to attain low energy consumption. The beacon-enabled mode behavior is analyzed in [38] to define the main performance bottlenecks. The adaptation method is referred to as $\mathrm{ABE}$ where a coordinator observes the number of idle slots before a transmission starts. However, a node consumes energy to observe idle slots because it needs to be awake. This problem is solved by delegating tracking of idle slots to the coordinator that always needs to stay awake during the active period. $\mathrm{ABE}$ is compared with the IEEE 802.15.4 standard configured with static backoff exponent which specifies the size of the contention window values. Results show that $\mathrm{ABE}$ provides optimal throughput without considering the number of nodes. The value of the contention window which optimizes throughput is found by the coordinator in $\mathrm{ABE}$. The adaptation mechanism in ABE which adjusts the optimal contention window values yields high throughputs along with low duty cycles, leading to low energy dissipation.

Forcing the nodes to sleep adaptively while ensuring the continuation of communication is the purpose of [39]. The Base Station Controlled MAC (BSC-MAC) conserves energy by switching off the idle nodes. The source nodes generate the data and forward it to the base station through the root nodes. In BSC-MAC, the list of all nodes reaches the base station by flooding. The nodes with subsets are detected, and they are designated as the root nodes. The capture of a path causes the neighbor nodes to go to sleep mode. This mechanism provides power savings, reduces packet collisions, inhibits packet losses, and also prolongs the network lifetime. A NS-2 simulator is used for simulations. BSC-MAC is compared with Pattern Medium Access Control (P-MAC) which designates the sleep of sensor nodes adaptively, Timeout Medium Access Protocol (T-MAC) which is used for idle listening for wireless sensor networks (WSNs), and Sensor Medium Access Protocol (S-MAC) which puts the nodes into sleep mode periodically when the nodes unnecessarily listen to the environment to the point of total energy consumption for all scenarios. BSC-MAC performs better than S-MAC. It has worse results than P-MAC when the total number of generated packets increases. Also, BSC-MAC is compared with Adaptive Energy-Efficient MAC (AEE-MAC) which provides more energy efficiency than MAC by using additional improvements: adaptive sleep, reuse of the channel, and combined control packets. BSC-MAC can result in more energy savings compared with AEE-MAC.

Various MAC schemes have been proposed to enhance resource sharing and energy preservation for WSNs. However, these MAC mechanisms are complex and expensive. For this reason, simulators are required to understand the network behavior. The authors in [40] are interested in the performance of wireless MAC protocols for energy dissipation. A simulator (EnergySim) is developed to evaluate energy consumption of a network and nodes for any given wireless MAC protocol. 
EnergySim provides position information of the nodes, nodes' residual energy, energy consumption, and the number of alive nodes. It is an event-driven simulator and specifies the shortest paths by the usage of the Dijkstra algorithm. It allows to make detailed energy analyses for any given wireless MAC protocol. Table 2 compares some CSMA/CA-based MAC protocols for their energy efficiency.

\section{LTE (Long-Term Evolution)}

Wireless access networks consume $80 \%$ of the total energy used by mobile communication network operators. Radio resource management and allocation functions for LTE have become an important research subject as energy consumption in base stations and downlink transmissions seems to be a major area where significant conservation can be achieved.

Instead of optimizing to improve spectral efficiency of access networks, in [41], energy efficiency of some wellknown principal scheduling schemes is introduced. Three relevant energy-efficient schedulers are introduced: round robin (RR), best channel quality indicator (BCQI), and proportional fair (PF). RR is the one of the most popular principal schedulers. It assigns the same number of physical resource blocks (PRBs). BCQI and PF are channelaware scheduling schemes. BCQI selects the user with the highest channel quality index (CQI), which measures the quality of a wireless communication channel; in other words, a high CQI is indicative of a high-quality signal at each transmission time interval (TTI) for each PRB. In $\mathrm{PF}$, the users are selected based on a normalized achievable rate at each time slot. When the energy consumption model is examined, a linear power consumption model is considered. The bandwidth is traded off for energy whenever possible. According to derivations, the amount of energy consumed in each TTI is reduced so it provides overall reduction of the transmission energy. Finally, a maximum of one PRB is assigned to each user, and three energy-efficient schedulers are obtained: energy-efficient round robin (EERR), energy-efficient proportional fair (EEPF), and energy-efficient best channel quality indicator (EEBCQI). In simulations, a network with a single base station with multiple users is used. The performance of the proposed schedulers (EERR, EEPF, EEBCQI) is compared with that of principal schedulers (RR, PF, BCQI) in terms of energy efficiency and QoS. According to results, when there is a low number of available resources (PRBs) in the system, the proposed schedulers save significantly more energy than the principal schedulers. By increasing the number of PRBs, this energy gain scales up because all of the PRBs are not occupied as ON/OFF traffic source is considered. So, if all the PRBs are assigned to the users, the energy gain will rise. In conclusion, this work shows that energy savings gain mostly depends on the available resources (PRBs) and number of users in each TTI.

3GPP Long-Term Evolution-Advanced (LTE-A) is one of the advanced technologies for mobile communications. In LTE-A, multiple QoS classes have been defined for different bit rate, packet delay, and packet loss rate characteristics. In order to decrease the power consumption of the user equipments (UEs), a discontinuous reception (DRX) mechanism has been designed in LTE-A. This mechanism switches off the radio interfaces of the UEs when evolved node $B$ (eNB) does not have data to deliver to the UEs. Existing studies show that the usage of DRX can significantly reduce energy consumption in UEs. Although these studies have mentioned the DRX optimization problem, they did not consider QoS requirements and aimed to manage the DRX cycle length for UEs to enhance energy efficiency. Authors in [42] mention the DRX optimization problem. This study provides QoS satisfaction for UEs and saves energy by minimizing wake-up periods of UEs. A packet scheduling method and an efficient scheme are proposed. The proposed scheme is compared with the counter-driven DRX (CDD) which manages the length of the DRX cycle for each UE by using predefined thresholds, and multiple-threshold DRX (MTD) which designs multiple lengths and thresholds. The effects of packet loss rate, rate satisfaction ratio, and power consumption are examined. Reduced packet loss rates are obtained by using the proposed scheme since it extends wake-up periods and catches more packets. The proposed scheme possesses the highest satisfaction ratio when the network is saturated. Additionally, the power consumption performance of the proposed scheme has been observed to be in between the MTD and CDD. CDD outperforms the proposed scheme

Table 2 Comparison of MAC protocols

\begin{tabular}{|c|c|c|c|c|}
\hline Protocols & Type & Latency & Throughput & Energy efficiency \\
\hline P-MAC & Contention based & Medium & Medium & High \\
\hline T-MAC & Contention based & High & Low & High \\
\hline S-MAC & Contention based & Low & Low & Medium \\
\hline AEE-MAC & Contention based & Low & Low & Medium/high \\
\hline BSC-MAC & Contention based & Low & Medium & Medium/high \\
\hline
\end{tabular}


in terms of energy efficiency; however, it neglects the QoS satisfaction of UEs.

While some studies aimed to decrease the energy consumption of UEs in mobile networks, UE energy consumption is only a small fraction of the energy consumption in these networks. A larger fraction belongs to BSs. A study [43] is developed as part of the EARTH Project which aims to improve energy efficiency of LTE cellular networks while providing more wide coverage and to reduce the carbon footprint. Authors aim to enhance radio transmission techniques such as beamforming and MIMO antenna techniques to promote the objectives of the EARTH Project. Especially MIMO is used to enhance the spectral efficiency of the communication links. It is pointed out that in macrocells, significant reduction in power consumption can be obtained at high loads by using reconfigurable antennas. A suitable MIMO mode selection and proper precoding and scheduling can provide even more power savings. Also, significant power reductions are attained in picocells with convenient MIMO mode selection.

In [44], the design comparison between LTE and LTE$A$ is presented in terms of energy efficiency. LTE-A adds three new functionalities: carrier aggregation which increases the bit rate, heterogeneous networks which include combination of macrocell- and femtocell-based stations, and having multiple antennas in other words improved MIMO support to LTE. The energy efficiency in wireless access networks is formulated by specifying the most energy-efficient BS. The effects of the three new functionalities of the LTE-A on energy efficiency are investigated. The results show that especially LTE-A's carrier aggregation and MIMO enhancement provide significant increment on energy efficiency when compared with LTE. LTE-A also provides improved support for relaying, and this can also improve energy efficiency even more.

\section{Network layer}

The energy consumption of the ICT sector has grown rapidly in the recent years with the exponential increase in the number of people and devices that are connected to the network as well as the broad number protocols that have become key components of our daily lives. As a result, energy-efficient solutions have become a major concern and scientists have started to work on energyaware protocols. With the next generation of networking, we will soon be using energy-efficient protocols which will replace the current protocols developed without any energy constraints.

Data centers are one of the largest and fastest growing energy consumers in the world as they have become the backbone of many institutions. The explosion of Internet traffic and its content pushes data centers' energy needs forward. Thus, a significant amount of effort is spent on designing energy-efficient data centers. Switching off existing devices when they are idle is also an important candidate for providing an immediate energyefficient solution. However, the questions of when and how to make these decisions without an impact on the performance need to be answered. There are also energy efficiency studies on optical IP, mobile ad hoc networks, and wireless sensor networks. In this section, we try to describe some recent energy-efficient approaches for the network layer.

\section{Switching off network devices}

Switching off lightly loaded devices such as network links and/or nodes is one of the extensively studied energy efficiency methods as shown in Figure 3. Switching off network devices can result in immediate and significant energy savings provided that it does not have a negative impact on the network traffic. For this reason, traditionally used protocols need to be enhanced to compensate for switching off solutions.

With the Open Shortest Path First (OSPF) protocol, shortest path trees (SPTs) are computed and used by all routers in the network. Energy-Aware Routing (EAR) [45], on the other hand, uses only a subset of router SPTs for traffic routing. The EAR protocol classifies the routers into exporter, importer, and neutral routers. The exporters calculate their SPTs, and then the importers use these SPTs to construct their own modified path trees (MPTs) and to determine the links that can be switched off. Neutral routers act as in the traditional OSPF routing protocol. The performance of the protocol is measured by a using a performance index which shows the the percentage of links that the EAR protocol allows to switch off. The simulation results indicate that a significant number of links can be switched off and energy savings can be increased especially under light traffic conditions.

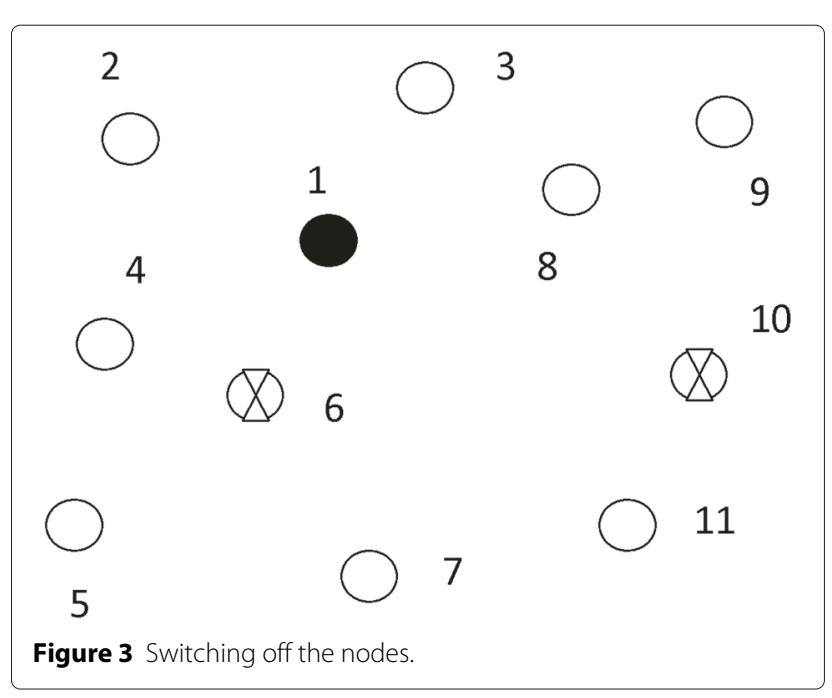


In [46], a distributed link management algorithm for energy-efficient IP networks is developed. The links in a network can be switched on/off based on the dynamically managed two threshold levels. The threshold levels are chosen based on the link flow amount. The distributed link management algorithm provides redirecting the flow on the fly without losses or loops during the long convergence of the routing protocols after switching on/off the links. In this work, the authors also implement an optimization tool which can specify the optimal power saving network topology.

A distributed approach [47] utilizes sleep mode abilities of links in an ISP network with the knowledge of current link load instead of current traffic matrix. The working states of the links are adjusted dynamically, and the network capacity is adapted to the current traffic load without overloading the resources. The optimization is to minimize the network power consumption under specific traffic conditions and QoS limitations. Distributed Least Flow (DLF) which selects the least loaded link and Distributed Most Power (DMP) which selects the most power-hungry link is used for algorithm design. These two algorithms are further divided into LastSleep which selects the last link entering a sleep state and Distance which selects the closer sleeping link to the congestion point. According to results, the DMP algorithms are more powerful than the DLF in terms of sleep attempts because DMP aims energy-hungry links which carry a lot of traffic. Furthermore, Distance policies save more power than LastSleep policies.

Minimizing energy consumption by putting idle routers and links into sleep mode and, furthermore, keeping the interference on network operation minimal is studied in [48]. On the contrary to the previously discussed works, this work can put both nodes and links into sleep mode. The optimization problem is formulated to maximize the number of sleeping nodes and links by taking into consideration the load balancing and the link utilization variation problems, and a heuristic Energy-Efficient Topology (EET) protocol is proposed. In the derivations, the energy efficiency of the nodes and the links is expressed separately, and the average energy efficiency of the overall network is obtained. The subset of routers and links that can be put into sleep mode is found. It is pointed out that more energy can be saved by switching off a network node than putting a single link into sleep mode. Thus, in EET protocol, the maximum number of nodes that can be switched off is determined first and then the links that can be switched off are identified. When the network load is low, a larger amount of routers can be selected to put into sleep mode.

A table lookup bypass (TLB) approach is proposed in [49]. Under suitable traffic conditions, the table lookup (TL) operation of a line card (LC) is bypassed and the received packets are deterministically forwarded through the next-hop router. Forwarding engine (FE) functionalities are frozen, and power dissipation is significantly reduced. By this way, the forwarding operation is independently achieved by each LC in core IP routers. The proposed approach also combines TLB and link switch off (LSO) approaches. There are two alternatives: to switch a whole link off or freeze out only the FE of LC. Especially in high traffic conditions, the proposed approach promises a lower energy consumption. It is pointed out that TLB is very suitable when LSO cannot be fulfilled; in other words, traffic load does not allow switching off of other links when the network is highly meshed.

In [50], a novel energy-efficient routing approach called Safe and Practical Energy-Efficient Detour routing (SPEED) is proposed. It provides power saving maximization with guaranteed connectivity by applying two key ideas: precomputing loop-free next-hops for each primary next-hop to forward traffic and selecting loop-free further next-hops then aggregating traffic to them without extra routing costs. Primarily, the proposed approach achieves energy savings by putting idle links into sleep mode after traffic aggregation. In simulations, real network topologies GEANT and Abilene are used. The performances of ORIG (routings computed by a traditional intradomain routing protocol), SPEED (proposed protocol), SPEED-C (proposed protocol with modified network configurations where the link weights are modified), OPT-MAX (the traffic over links with heavier loads will be pruned first), and OPT-MIN (traffic over links with lighter loads will be pruned first) are compared. According to the power saving ratio analyses of the simulations, it is proved that SPEED and SPEED-C are efficient for power savings in the traditional IP networks.

An energy efficiency and load balancing optimization algorithm called as Green-Load-balancing Algorithm (GLA) based on existing link switch off techniques is introduced in [51]. By optimizing the Interior Gateway Protocol (IGP) link weights of a network, energy saving gains are obtained by using the link sleeping and load balancing jointly. The algorithm aims to put more links to sleep without affecting the load balancing requirements. In simulations, the GEANT is used. The GLA is compared with Interior Gateway Protocol Weight Optimization (IGP-WO), and it is obtained that GLA shows slightly better performance than IGP-WO in terms of load balancing and results in energy savings at the same time. According to several experiment scenarios, the proposed algorithm improves the energy efficiency and enhances the performance of existing switch off algorithms in terms of energy efficiency.

A distributed energy-aware traffic engineering analysis DAISIES is proposed in [52]. DAISIES uses routing-based approach where the link switch off process is handled 
individually by each node. The switching off process is managed with respect to the traffic variations. A definite load-dependent link-weight function is proposed to perform DAISIES more efficiently. The proposed scheme is different from other studies in two important aspects: there is no off-line centralized component, and it searches for the best path for each traffic demand rather than search for the best set of links to switch off. It takes advantage of traffic engineering of the existing routers and modifies the path computation strategy, and it does not require significant changes in current routers. The event-driven simulation packet is used for simulations. For different traffic conditions, DAISIES performance is tested and it is observed that the proposed scheme provides significant energy gains.

In [53], switching off links and routers, managing the link weights for minimizing the energy consumption besides the network congestion with priority order is aimed. A Mixed Integer Linear Programming-based algorithm for Energy-aware Weights Optimization (MILPEWO) which takes advantage of the IGP-WO algorithm is presented, and the link weight optimization is configured. Link capacities are considered, and the network congestion level is minimized for ensuring service quality. According to the simulation results for several real network topologies, significant energy savings can be gained by using the efficient configuration of link weights in IP networks with homogeneous and heterogeneous network equipments by switching off a considerable number of core nodes.

In [54], the green routing algorithms are analyzed based on realistic network scenarios. idleEnergy, fully proportional, and energy-agnostic models for a network device energy consumption are investigated with an objective to find the network configuration that minimizes the total energy consumption. The pan-European data network GEANT is used for comparing Green and IGP-WO routing algorithms for idleEnergy (realistic case), fully proportional (best case), and energy-agnostic (worst case) energy models. The results conclude that on all energy models, it is possible to achieve higher energy savings by switching off links rather than by switching off nodes. In addition, it is observed that the energy saving performance depends on the network topology, traffic requests, and the device technology rather than the maximum device utilization.

\section{Optical IP}

Telecommunication networks can be classified into access, metro, and core domains. Core networks consume a significant amount of energy as they support high capacities with very high data rates. Core networks depend on optical transmission. In particular, the IP over wavelength-division multiplexing (WDM) technology is used for compensating increased Internet bandwidth requirements. For reducing the power consumption, the optical switching solutions are chosen. The most significant advantage of the optical switches is that their power dissipation does not increase significantly as the data rate increases. Generally, three types of optical switching solutions - optical circuit switching (OCS), optical burst switching (OBS), and optical packet switching (OPS) - are considered. In OCS, low costs and power consumption reductions are proposed; however, flexibility and bandwidth utilization is restricted. OBS sets up data into bursts and decreases network costs and power, but results in high data losses at core nodes. The highest flexibility and resource utilization is obtained by OPS, and it can be considered as the best alternative for electronic switching. However, it needs complex optical components that lead to high power consumption.

For exploiting the advantages of OCS, OBS, and OPS on the same network, the hybrid optical switching (HOS) is implemented in [55] for achieving an energy-efficient Internet core. Three possible architectures - an alloptical HOS network, an optical/electronic HOS network, and all-electronic network - are proposed for future high-capacity networks. For comparison of the proposed network solutions, the Pan-European network is used. According to the results, the all-electronic network produces the highest carbon dioxide emissions. As the input load in HOS networks is lower, the HOS network emissions as well as the energy consumption are lower. But it can cause a decrease in the network throughput and larger data loss rates.

In [56], adaptive networks and mixed-line rate (MLR) IP networks are compared over DWDM networks in terms of cost and power efficiency. With a realistic IP traffic pattern, the network optimization scheme optimizes the IP layer and WDM layer by setting additional IP links to bypass IP routers and route them through suitable transmission links except for all links that minimize the power consumption of the whole network. The simulation results show that the MLR network achieves more power gain than the adaptive network which is more hardware efficient in terms of total number of transponders with hierarchical scenario (higher link load but less number of IP links) and flat scenario (additional shortcuts between ex-region nodes together with lower link capacities) in both optimized IP layer and optimized WDM layer.

In [57], the power savings achieved by optimization of the physical topology of IP over WDM networks is investigated in terms of non-bypass and bypass approaches under nodal degree constraints, traffic symmetry, and renewable energy availability. The results of simulations show that the optimized physical topologies provide significant energy gains when compared to the NSFNET topology, which covers the US and provides a realistic scenario, delay-minimized topology, and the virtual topology. 
In [58], the energy consumption of IP over optical WDM networks is analyzed through an optical power-aware backbone network model. It is stated that the energy consumption of the IP over optical WDM networks can be reduced by bypassing the lightpath in the optical layer which causes a reduction in the number of IP router ports that consume maximum energy. In an energy-minimized design, the set of nodes corresponds to IP routers and optical switch nodes, and the link set symbolizes the physical fibers in the network. The proposed optimization model decreases energy dissipations in the network such as IP routers and WDM transponders to minimize overall network energy consumption. From the simulation results, it is observed that the proposed scheme consumes less power than the traffic grooming scheme when the lightpath bypass strategy is implemented. It is seen that the network size growth increases the power savings of the lightpath strategy because it provides longer lightpaths and allows bypassing of more intermediate switch nodes.

In [59], the energy efficiency of rate and modulation adaptive optical OFDM-based networks is investigated. OFDM promotes heterogeneous traffic demands. The distance-adaptive spectrum allocation which includes the adaptation of the modulation level of subcarriers by using optical paths of higher optical signal-to-noise ratios (OSNR) is taken into consideration with flexible bandwidth allocation. The proposed model differentiates the power-minimized and spectrum-minimized optimization schemes. In simulations, the optical OFDM-based networks are compared with conventional IP over WDM networks under the bypass approach. OFDM-based networks are also compared with MLR networks. The proposed model saves a significant amount of energy compared to IP over WDM networks and MLR networks. In addition, MLR networks also have higher energy efficiency than WDM networks.

\section{Data center networks}

Data centers are among the major contributors of energy consumption for the ICT sector due to the ever growing demand for video and data extreme applications in various fields such as informatics, genomics, finance, and medicine. To make things even worse, the cost of energy is steadily increasing. There are many studies that focus on minimizing the power consumption inside the data centers, as it is estimated that the data centers are responsible for approximately one quarter of the overall network power consumption. According to [60], the amount of energy consumed by data centers is growing by $12 \%$ per year. One data center can use enough electricity to power 180,000 homes; in other words, they can consume up to 100 times more energy than a standard office building [61]. In this section, we present recent studies that focus on decreasing power usage in data centers and reducing the power dissipation of data transportation between data centers and end users.

Air cooling represents an important share of the energy expenditure in data centers. If computational power, temperature, leakage, and cooling power needs can be reduced, major energy savings might be possible. In addition to air cooling, the location of data centers can have a significant impact on energy usage. For over 10 years, Google [62] has been building some of the most energy-efficient data centers in the world. Their data centers consume $50 \%$ less energy than the typical data centers, resulting in a savings of more than a billion dollars to date. By displacing the server farms to the banks of the Colombia River, the energy offered by the hydroelectric power plants nearby has been used with significant savings. The water flow provided by the river in addition is used within the cooling systems. An alternative cooling system designed by Microsoft in the Tent and Marlow projects [63] leaves servers in the open air so that heat can dissipate quickly.

Dong et al. [64] study the location of the data centers for minimizing the power consumption through mathematical optimization models. Two traffic scenarios: the traffic towards and from the data centers and the traffic between data centers with the non-bypass approach and the multi-hop bypass approach are analyzed. The optimal data center locations provide approximately one quarter more energy gain when compared to the worst locations in the non-bypass approach. The energy gain value is about one half of the non-bypass case with the multi-hop bypass approach. The problem of whether to locate data centers next to renewable energy or to transmit renewable energy to data centers is studied and concluded that a significant energy gain can be obtained in optimization of the data centers' locations with renewable energy.

As leakage power is exponentially dependent on temperature, higher temperatures can cause higher power consumption. Fan speeds affect the power dynamics of servers and can cause an increase in temperature and leakage power. In [65], a leakage and temperature-aware server control mechanism is presented for enhancing energy efficiency of enterprise servers in data centers. In order to determine the leakage-temperature effects on the energy efficiency of servers, two fan control schemes - the LUT-based Controller which periodically monitors the load utilization and provides fan speed management of the power supplies and the Bang-Bang Controller which keeps the temperature in between two desirable temperature values - are presented. According to the results, both fan control schemes provide energy savings in comparison to the original fan scheme. It is also observed that, the LUT-based scheme is better in energy efficiency because the runtime temperature values are lower and steadier and leakage is always kept low. 
A study for temperature power management in [66] tries to optimize temperature sensor deployment in data centers to control cooling supply which is still a problem in the data center energy efficiency perspective. To solve this problem, authors deploy sensors in a single rack and aim to extend the algorithm to the whole data center. Then, an optimization problem is formulated to determine proper positions for temperature sensors to adjust cooling supply. In simulations, the relationship between the optimal temperatures and optimal sensor positions is investigated. It is observed that significant energy savings can be obtained when compared with random sensor deployment.

Authors in [67] enhance techniques to analyze and optimize energy management in multi-core servers by involving the dynamic power of the processor in addition to leakage power and switching overhead between speed levels. An objective function that is a linear combination of energy usage, queuing cost, and switching costs is established. The considered processor is multicore where each core is running at a different speed, while previous studies considered only the case where all the cores were running at the same speed. According to the results, a significant part of the energy efficiency gain is obtained with one or two thresholds. Also, the hysteretic model, in which there are two threshold buffer levels and the queue is served with the lower service rate, outperforms the one-threshold model in terms of energy efficiency. Consequently, the most energy-efficient model is the two-threshold model for all scenarios.

A new throughput-guaranteed Power-Aware Routing algorithm (PRA) is presented in [68] which aims to reduce the total power consumption of network devices. PRA provides efficient power utilization in data center networks by exploiting sufficient network power to ensure the routing service while sustaining the target network throughput. In addition, the trade-off between power conservation and network fault tolerance is investigated. The proposed approach uses a different rate model where the transfer rate of each flow is bandwidth constrained and depends on network resource competition with other flows rather than the constant traffic demand. The proposed approach is pruning-based. The switches and the links are iteratively eliminated, and it is a significant alternative for existing routing algorithms. BCube and Fat-Tree topologies which are used for data center networks are considered in simulations. The results are obtained in the sense of various traffic patterns. It is obtained that the proposed algorithm decreases the power consumed by switches at low network loads, and as expected, the power saving percentage is inversely proportional to the network load. Besides, the proposed algorithm is compared with the ElasticTree scheme where every flow has a constant transfer rate. Results show that PRA obtains abundant power gains than ElasticTree.
Since networking, servers, and cooling are the major energy consumers in data center networks, most of the existing studies focus on energy-aware routing and topology designs rather than scheduling traffic flows in a more energy-efficient scheme. In [69], authors depart from this idea and propose a joint manner which combines preemptive flow scheduling and energy-aware routing. In the proposed algorithm, Flow Preemption and EnergyAware Routing (FP and EAR), the higher priority owner flow is preferably scheduled and the lower ones are suspended until the flow ends. This scheme makes the links completely utilized and saves more energy. Then, the energy-aware routing assigns the paths sharing switches sufficiently to the traffic flows which are scheduled at the same time in the network. In the simulations, the performance of the FP and EAR is compared with ECMP routing which is traditionally used in data center network topologies and achieves load balance and high network performance. According to results, the network energy is saved significantly by the proposed algorithm under the impact of flow arrival interval. In addition, the effect of traffic flow distribution on the network energy consumption is also evaluated. It is seen that when the percentage of used servers is low, the impact of FP and EAR increases significantly.

\section{Mobile ad hoc networks}

Unreliable wireless links and multiple receivers associated with a single packet transmission can result in poor energy efficiencies for wireless networks. In this section, several studies aiming to overcome these difficulties and providing for energy-efficient solutions for mobile ad hoc networks (MANETs) are described.

The performance of several routing protocols such as AODV, DSR, DSDV, TORA, and EAODV for MANETs is compared in existence of frequent link failures due to the high mobility of the nodes in [70]. In energy efficiency analyses, it is pointed out that for measuring energy efficiency, the network lifetime can be used instead of power consumption. In simulations, four performance metrics - packet delivery ratio (PDR), normalized routing load (NRL), average end-to-end delay, and energy consumption - are used. Performance results of these metrics are obtained with the impact of three parameters: node velocity, packet sending rate, and pause time. The energy consumption of the on-demand protocols increases as the maximum node velocity increases. The results show that DSR has lower energy per user data than EAODV and DSDV. When velocity increases, the energy efficiency of the DSR is better than EAODV, TORA, and DSDV.

A scalable, opportunistic, and energy-efficient routing procotol (E2R) is proposed in [71] which employs a greedy forwarding algorithm by reducing the control message overhead. E2R protocol is highly distributed and scalable 
as it requires one-hop neighbor information. The protocol exploits spatial diversity in wireless networks rather than determine data delivery paths such as next hop. Thus, the discovery packets and data packets are delivered by broadcasting. The proposed protocol is compared with AODV and SBA-AODV (scalable broadcast algorithm AODV). In SBA, before rebroadcasting the first received packet, each node waits for a randomly assigned time interval. Packet delivery ratio, control overhead, and packet delivery delay are the metrics used for evaluating the protocols. The impact of node density and node failure is examined for these metrics, and it is observed that the control overhead is $90 \%$ reduced compared to AODV.

The energy-efficient routing protocols are generally classified into two categories in literature: Minimum Energy Routing Protocols and Maximum Network Lifetime Routing Protocols. Progressive Energy-Efficient Routing (PEER) presented in [72] is a minimum energy routing protocol. Contrary to other energy-efficient routing protocols that try to find the optimal path during route discovery process, PEER searches for the more energyefficient path progressively and ensures the route continuously. A suboptimal path to the most energy-efficient path is formed between the source and the destination, and the transmission path changes when necessary with slight overhead to guarantee energy-efficient transmissions. PEER is a cost-based protocol. The cost of all the links are calculated, and the minimum cost path is selected. In simulations, the performances of PEER, Minimum Total Reliable Transmission Power (MTRTP), and AODV are compared in static and mobile scenarios. In the static scenario, PEER performs the best in terms of energy consumption per packet followed by MTRTP and AODV. PEER performs better than MTRTP because it uses more exact link costs, its routing overhead is lower, and it can adapt paths to the environment changes quickly. Also, PEER and MTRTP perform better than AODV as they search for the most energy-efficient path instead of the shortest path. In the mobile scenario, PEER has the best and MTRTP has the worst performance as MTRTP cannot adjust well to mobility. Consequently, PEER reduces energy consumption by approximately $25 \%$ compared to AODV and 40\% compared to MTRTP in the mobile scenario.

In MANETs, nodes may act selfishly as the network does not rely on a pre-existing infrastructure. Topology control game is examined in [73] for attaining an energy-efficient topology in MANETs in the existence of selfish nodes. The proposed scheme uses the benefit of a node for joining a network as a function of its transmission power. However, the energy consumption of the nodes also depends on the volume of transferred traffic. A new objective function which takes into account both load and transmission power is described. For evaluating the energy efficiency of the communications, the energy usage of nodes is calculated. For global methods, each node is aware of the whole information about the connectivity of all other nodes. On the other hand, every node is only aware of topological information about nodes within a limited hop distance in local methods. Significant energy efficiency is obtained when each node merely removes one link. Local methods outperform the global methods in terms of energy efficiency.

A wireless mesh network (WMN) is a particular type of MANET which is reliable, offers redundancy, and consists of radio nodes organized in a mesh topology. It often includes mesh clients such as laptops, cell phones, mesh routers, and gateways. A routing algorithm based on Strength Pareto Evolutionary Algorithm (SPEA) is proposed in [74] for WMNs. SPEA aims to build the most efficient routes. These routes consider the shortest path, energy consumption, and QoS limitations. The main objective of the proposed protocol is to find an optimal route, which is energy efficient, by decreasing the possibility of packet loss and satisfying the maximum delay and minimum bandwidth requirements of the connection, for unicast or multicast schemes. In the work, a mathematical model is described with four objective functions: hop count, energy consumption, free space loss, and delay. For the energy consumption function, it is assumed that the network is formed by heterogeneous nodes and they can have different energy consumption for both transmission and reception. The proposed protocol selects the path with the lower overall energy consumption to increase the battery life of these devices. In simulations, different scenarios are configured to compare the solutions of the proposed algorithm and the traditional shortest path algorithm. When the power consumption is individually minimized by minimizing the energy consumption function, significant energy gain is achieved.

Delay-tolerant networks involve the technical issues of heterogeneous networks and are deprived of continuous network connectivity. They can operate in mobile or extreme terrestrial environments. Energy-efficient Routing and Rate Allocation (ERRA) protocol for a delaytolerant MANET is described in [75]. The existing routing schemes for delay-tolerant networks (DTNs) mainly utilize metrics such as delay, hop count, and bandwidth. ERRA is designed for optimizing the energy efficiency with constraints of congestion, buffer, and delay bound for DTNs. In traditional networks, three events can occur: listening, sending, and receiving. In ERRA, if no events occur at a node, the node can switch to sleep state for saving energy. In energy consumption analyses, the energy cost function is calculated by considering the average amount of energy consumption per bit. In simulations, an ad hoc delay-tolerant network is used. A fixed static node is placed at the center and used as an access point. ERRA is 
compared with the Epidemic and modified Dijkstra algorithm. Epidemic routing uses a naive flooding method, but it wastes resources and reduces the network performance. The modified Dijkstra algorithm is based on a time-dependent graph forwarding scheme and maintains at most one copy of a packet in the network during the routing process. ERRA provides better energy efficiency because the Epidemic algorithm uses copy to deliver data packets so more energy is consumed. The modified Dijkstra algorithm uses forward method for minimizing the average delivery delay, but it does not consider energy wastings.

\section{Wireless sensor networks}

WSNs consist of distributed sensors which monitor physical or environmental properties, such as temperature, humidity, and pressure, and pass the collected data to a sink node. WSNs have a wide range of usability in many distinct areas, such as science, transportation, habitat, emergency, and military applications. The primary challenges in a WSN include energy efficiency, responsiveness, robustness, adaptation, and self-configuration. Similar to MAC protocols described before, routing protocols also have a significant impact on the energy consumption of WSNs. In this section, energy-efficient routing approaches for WSNs are discussed.

Limited energy availability in sensor nodes makes network lifetime an important issue in WSN applications. To extend the network lifetime, energy-efficient wireless sensor network protocols and algorithms have been devised in the literature. Node clustering, in-network data processing, data fusion, and network coding are some of the measures taken to reduce the amount of data that is processed, sensed, or transmitted. Minimization of energy spent in processing, sensing, and transmission of data allows sensor nodes to save energy. Such energy savings help to extend the lifetime of WSN applications.

The main goal of cluster-based routing protocol is to efficiently maintain the energy consumption of sensor nodes by involving them in multi-hop communication within a cluster and by performing data aggregation and fusion in order to decrease the number of transmitted messages to the sink and transmission distance of sensor nodes. Low-energy adaptive clustering hierarchy (LEACH) [76] forms clusters randomly in an adaptive and self-organized way. Figure 4 shows an example of a random cluster formation and cluster head $(\mathrm{CH})$ selection with LEACH.

The LEACH protocol allows clusters and $\mathrm{CH}$ s to change dynamically based on the energy levels of the $\mathrm{CHs}$, and Figure 5 shows another possibility for the network configuration with $\mathrm{LEACH}$.

LEACH provides a significant decrease in the overall energy consumption of the network and improves network lifetime and decreases number of dead nodes. The randomly determined cluster formations of LEACH especially prolong the lifetime of the remote nodes to the base station (BS). Relay or route nodes are usually very energy harvesting, but changing the clusters and $\mathrm{CHs}$ in each round can extend the lifetime of relay and route nodes.

On the other hand, LEACH has some disadvantages. The random determination of clusters and $\mathrm{CHs}$ can

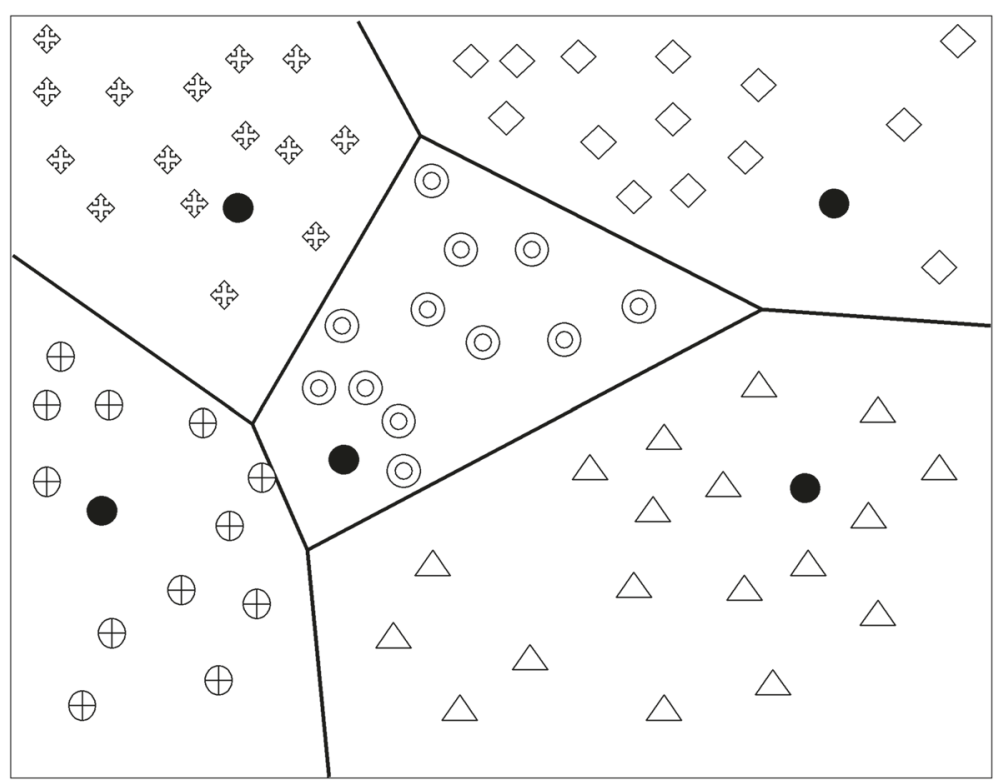

Figure 4 Cluster formations in LEACH, the cluster formation in the existing round. 


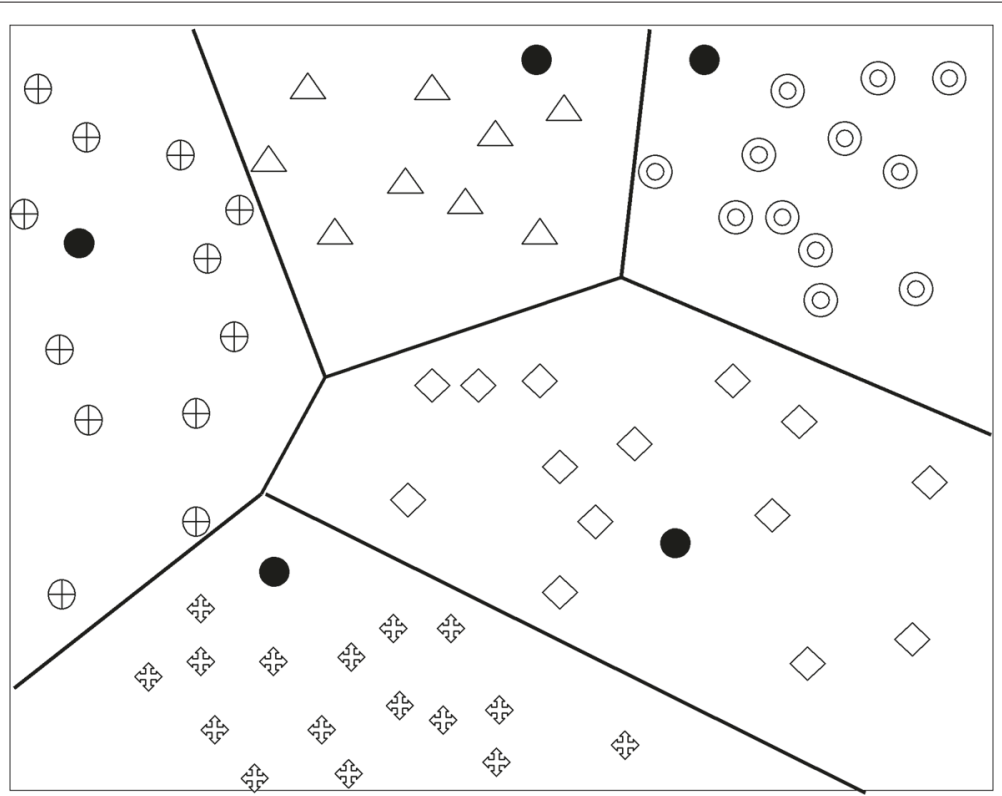

Figure 5 Cluster formations in $\mathrm{LEACH}$, the cluster formation in the next round.

cause inefficient cluster formations and unbalanced cluster sizes. In some cases, the position of $\mathrm{CHs}$ cannot be optimal and they can be very far from the BS; thus, their batteries drain quickly and they can die prematurely, and hence, this can shorten the network lifetime. Also, the dynamic configuration of frequent cluster formations and $\mathrm{CHs}$ can induce more energy dissipation and can bring extra network costs while broadcasting the advertisement messages of the $\mathrm{CHs}$. In recent years, numerous studies are proposed to solve the problems of LEACH and to improve its performance, such as MR-LEACH [77], I-LEACH [78], LEACH-IMP [79], V-LEACH [80], and CLEACH [81]. Table 3 compares these protocols for their energy efficiency and lifetime.

In [82], an energy-efficient secure routing protocol for WSNs is presented. An integrated protocol which simultaneously takes into consideration the security and routing is proposed. The proposed protocol has three benefits. First, in order to offer a better delivery ratio, the protocol uses a sink-oriented grid structure originated from the grid design in Two-Tier Data Dissemination (TTDD) protocol. This protocol is a query-based routing protocol and designed to address the scalability and efficiency problem with multiple mobile sinks. Second, the benefit is that the proposed protocol searches the outermost highest energy emission node to find an efficient path from the source to the sink, resulting in a lower energy consumption. The last benefit is the security consideration. In the network model, a WSN with a stationary sink and stationary sensor nodes is considered. Without using GPS, each node knows its own position by using a localization discovery system. The nodes are homogeneous and conscious of their neighbors. From the source to the sink, multi-hop forwarding is used for data forwarding. In simulations, the comparison between the proposed protocol and TTDD is presented. According to results, the proposed protocol achieves significant improvement in delivery ratio because of the usage of the sink-oriented grid mechanism which is more

Table 3 Comparison of LEACH protocols

\begin{tabular}{|c|c|c|c|c|c|c|}
\hline & \multicolumn{6}{|c|}{ Protocols } \\
\hline & LEACH & MR-LEACH & I-LEACH & LEACH-IMP & U-LEACH & C-LEACH \\
\hline Cluster formation & Each round & Each round & Each round & Fixed & Each round & Threshold \\
\hline Self-organized & Yes & No & No & No & No & Yes \\
\hline Location usage & No & Yes & Yes & Yes & Yes & No \\
\hline $\mathrm{CH}$ selection & Random & By BS & Residual energy & Only once & Residual energy & Threshold \\
\hline Energy efficiency & High & Very high & Very high & Very high & Very high & Very high \\
\hline Network lifetime & High & Very high & Very high & High & Very high & Very high \\
\hline
\end{tabular}


successful in availability of the path from any node to the sink than in TTDD. The average energy consumption of TTDD is higher than that of the proposed algorithm, as in TTDD, the packet from the same source-destination pair travels through a longer path to the sink. The average hops in the proposed algorithm are relatively steady because of the estimation of the position of the sink through the farthest-highest energy dissemination node search, while in TTDD, they slightly increase with the node density. The longer the routed path, the more energy is consumed for transmission so the proposed algorithm is more successful than TTDD in energy conservation idea. Finally, in lifetime analysis, the proposed protocol provides significant improvement as compared to TTDD.

In [83], using the WSN, an energy-efficient routing approach in a VoIP network infrastructure is studied. Six steps - registering all mobile devices, arranging successful VoIP communication between mobile devices, clustering based algorithm, enhanced cluster head selection, registering mobile devices to their respective cluster heads, and selecting alternative cluster head - are defined. The outcome of the study shows energy efficiency in VoIP communication by using the clustering approach.

Pantazis et al. [84] provide a comprehensive survey for energy-efficient routing protocols in WSNs. The energyefficient routing protocols are classified into four main types: network structure (flat, hierarchical), communication model (query, coherent, and negotiation based), topology based (location and mobile agent based), and reliable routing (multipath and QoS based). The performance comparisons between these types are presented in terms of energy efficiency. For small networks with stable nodes, the flat protocols are suitable. For large networks, hierarchical protocols solve the incommodiousness of the flat ones and provide efficiency by dividing the network into clusters. The location-based and mobile agent-based protocols can be a proper solution for highly dynamic networks. The negotiation-based ones provide optimum performance in both point-to-point and broadcast networks in terms of energy gains. Multipathbased protocols sustain multiple paths from nodes to sink; however, they suffer from the overhead of table and state maintanence. QoS protocols solve the delivery accuracy problem of the data. Query-based protocols can be used for dynamic network topologies, and they also promote multiple route replies. Finally, the coherentbased routing protocols include energy-efficient mechanisms and provide minimum processing cost to the sensor nodes.

\section{Transport layer}

The transport layer provides end-to-end reliable/ unreliable communication service for the applications. The energy efficiency studies in the transport layer focus on increasing energy efficiency through new or enhanced transport layer protocols, but more commonly on TCP.

Authors in [85] investigate the effects of dynamic transmission control on the performance of the TCP. Measurement-based slow start (MSS) calculates the round trip time (RTT) to detect congestion and promptly enters into the congestion avoidance mode after finishing the slow start. The number of active links between switches is dynamically controlled by dynamic link aggregation protocol (dynamic LACP). MSS saves more power compared to compound TCP (CTCP) because the number of active links in MSS is less than that in CTCP and the available bandwidth is not used effectively in CTCP.

MPTCP is a coupled multipath-aware congestion control, and it proposes the resource pooling principle and provides a load balancing mechanism by moving more data traffic load into the less congested paths. MPTCP expands the standard TCP and allows data streams to be delivered across multiple connections and paths. This feature of MPTCP makes it quite charming for the recent consumer mobile devices that support multiple radio interfaces such as $3 \mathrm{G}, \mathrm{WiFi}$, Bluetooth, and LTE. However, an efficient multipath congestion control algorithm design that is both energy-aware and able to handle load balancing has not been addressed.

In [86], an energy-aware congestion control algorithm (ecMTCP) for multipath TCP is developed. In ecMTCP, the rate control depends on a traffic sharing principle between the paths and applied by its energy costs and traffic loads. The sender and receiver measure the energy costs of data and ACK packets, respectively. The energy costs of the receiver side is piggybacked to the sender periodically with ACKs, and finally, the sender calculates the overall end-to-end energy cost of the successful transmission of one data packet. Energy efficiency is provided by offloading traffic from higher energy cost paths to the lower paths and by using an energy-aware control mechanism where the congestion window increase is inversely proportional to the energy cost on each path. In simulations, energy efficiency is indicated by measuring the energy consumption difference of the proposed algorithm and MPTCP. According to results, the energy savings of ecMTCP is better than that of MPTCP.

Chen et al. [87] propose a novel energy-aware MPTCPbased content delivery scheme (eMTCP). eMTCP follows the channel status for each of the transmission paths and offloads traffic from the most energy-consuming interface to other interfaces for obtaining energy efficiency. In simulations, LTE and WiFi are considered. Data transmission performance is measured in terms of remaining energy, throughput, energy efficiency, estimated battery lifespan, and user-perceived quality. The performance of the eMTCP is compared with that of MPTCP and TCP. According to results, eMTCP achieves significant 
improvement on the battery lifespan when compared with the original MPTCP. Higher energy savings are achieved when compared with MPTCP and TCP.

Various versions of TCP such as AIMP, Cubic, Bic, Vegas, and CTCP are studied to analyze the congestion window and average throughput values in [88]. The ESTOP algorithm is used for decreasing the congestion window value. ESTOP switches off links in increasing order of an associated centrality level of the network by counting the number of shortest paths. In the algorithm, the switch off count is associated with a threshold value which preserves the network connectivity. After switch off operation, the link state update of OSPF is used for changing the topology. The ESTOP is simulated in the Abilene network. In simulations, a TCP flow is established between two distant nodes in the network. For the worst case scenario, the outermost two nodes are selected as TCP flow endpoints, and in these nodes, TCP flow is also affected in terms of available capacity after the links switch off. It is observed that, for the considered TCP versions, it is possible to gain energy in the network without a drastic compromise of the TCP performance. The switching off links obtained by using ESTOP produce higher energy savings.

The rapid growth of wireless Internet and broadband networking infrastructures, such as 3G, WLAN, WLANmesh, and WiMAX, makes multimedia information available anytime, anywhere, and on any device. An efficient transport protocol which reduces all types of losses by satisfying multimedia constraints is designed in [89]. Loss proportion increase and loss proportion decrease (PIPD) algorithm is used for defining burst period in order to increase efficiency. Efficient Transport Protocol (ETP) is implemented with NS2 to evaluate its performance over wireless Internet. Throughput, energy efficiency, fairness index, sending rate, data received with time, bandwidth, packets lost, and end-to-end delay are the performance evaluation parameters. ETP is compared with TCP Friendly Rate Control Protocol (TFRC), and it is observed that ETP performs better than TFRC for energy efficiency.

An energy-aware mechanism for the TCP-based transport protocol is proposed in [90]. The proposed mechanism aims to enhance energy savings at the end hosts by decreasing the multimedia traffic amount through handling loss tolerance of multimedia applications. This tolerance is utilized by reducing the offered QoS to the minimum required by the application. Three end host interfaces - IEEE 802.3 (for wired networks), IEEE802.11 (for wireless networks), and UMTS (for 3 G mobile phones) are considered. When the network is more reliable, the proposed mechanism is more efficient. The number of generated packets is reduced, energy savings are achieved, and the minimal QoS requirements are provided.
A Reliable and Energy-Efficient Transport Protocol (REETP) is developed in [91]. The proposed protocol implements an efficient node selection algorithm which determines a set of efficient nodes called as E-Nodes. These nodes form a near-optimal coverage set with largest area and highest discard energy level. REETP transfers encoded packets using Luby Transform (LT) Code from the source to the sink block by block, and each of these blocks is forwarded to an E-Node. After the reception of the encoded packets, the E-Node tries to reconstruct the original data packets and it encodes the original data packets again and relays them to the next E-Node until it reaches the sink. The results are compared with MAC-aware Energy Efficient Reliable Transport Protocol (MAEERTP) which provides the desired event reliability to the application by distributing the load at a sensor. The energy-related results are better than MAEERTP. The results show that the proposed protocol has higher packet delivery ratio with reduced packet loss and energy consumption than MAEERTP.

Based on the data reliability notion, the authors in [92] propose a Data-Reliable Energy-Efficient Transport Layer Protocol (DREET). As sensor data varies slightly in relatively short times unless certain events occur, a small subset of sensor data can be constructed as key data and provide reliable transport only for these key data instead of the whole sensor data. Their solution decreases retransmission and energy while keeping data reliability. Simulation results show that DREET provides an optimal event detection rate and relative standard deviation (RSD) between sensor data and sink data, by introducing a tiny increase in energy consumption. It can be obtained from this study that,DREET is a novel transport layer solution for reliable event detection in WSN with minimum energy expenditure.

In [93], a rate-based transport protocol for reducing energy consumption of nodes in MANETs is presented. The percentage of power consumption time is considered as the evaluation parameter, and NS2 simulator is used. The simulations are realized for different numbers of nodes and for various data rates. The number of flows through the intermediate node is changed, and its impact is analyzed. A higher number of flows increases the load on the node as well as the power consumption. According to the simulation results, the proposed approach consumes less power than the Ad hoc Transport Protocol (ATP) where the packets are carried upward to the transport layer only to update the delay stamping, and the flow control is dealt with only at the end nodes.

\section{Application layer}

The application layer is the highest layer of the Internet protocol stack. It contains protocols for process to 
process communication, and it is the only layer where an end user directly interacts with. The actual communication between processes on end users is initiated in this layer. Although a great number of energy saving mechanisms whose impact might be significant are conducted on lower layers, we believe that the studies for this layer are also valuable.

Browsing a single web page can consume a significant amount of power, and this amount increases rapidly with the usage of applications involving streaming video. For example, Google has determined the power consumed during an average Google search in 2009. Three hundred million searches per day can cause $90,000 \mathrm{kWh}$ power consumption. The authors in [94] propose a model which supports energy-aware usage of web services. The proposed model estimates the power consumption during web service workloads. The factors which cause power consumption in web services such as nature of the $\mathrm{I} / \mathrm{O}$, server configuration, and web service design are investigated. In experiments, several measurable performance counters are determined, and several monitors are used. Multiple independent clients that invoke all of the web service operations are considered, and the samples of the actual power and performance counter measurements are collected. According to the results, a nonlinear model and CPU utilization-based power model segmentation increases accuracy. The proposed model significantly reduces the power consumption of the experimental web services and provides isolation of the power consumption of a particular web service within a regular server environment.

Mobile smart phones and other portable embedded systems such as PDAs and tablets are permeative devices. They are used for web browsing, multimedia, games, e-mail, and social networking. One of the most important parameters in mobile devices is battery lifetime. Some recent studies have discussed enhancing energy efficiency of applications in mobile environment, focusing on extending battery lifetime by using software optimizations. The authors in [95] present a novel application and user-interaction-aware energy management framework (AURA) for mobile devices. AURA exploits user idle time between interaction events of the spotlight application to optimize CPU and backlight energy consumption. It balances energy consumption and QoS demands. Based on user interaction, AURA dynamically classifies applications. After classification, the processor frequency and screen backlight levels are managed to reduce system energy consumption with Markov decision process-based power management algorithms. For user-device interaction field study, several Android applications from different categories are selected. In experiments, the performance of AURA middleware framework is compared with CHBL [96] which uses the changing blindness to increasingly ramp down CPU frequency and screen backlight levels over time. According to results, AURA presents higher energy savings because it dynamically adapts to the user interaction patterns and makes full use of user idle time. Contrary to this, CHBL is independent of user interaction patterns.

Another novel study for promoting energy-aware mobile application design is Anole [97]. It is a framework that aims to tell application designers the changing time of the energy consumption states of applications. The Anole framework involves three modules: energy profiler is a module used for estimating application energy consumption, energy-aware APIs that state applications by using the energy information and energy-aware policy which applies the energy adaptation of operating system by including service and hardware adaptations. The energy adaptation levels are predefined. This provides closing the services according to these levels. For example, when the level is six, the network service will be closed, and when the level is one, Bluetooth service will be turned off. The power consumption of the whole system is measured with voltage measurement of the resistor connected between the battery and mobile phone. To investigate the efficiency of application, energy adaptation, video player, and web browser applications are used and significant average energy savings are obtained.

In [98], user-aware streaming strategies for energyefficient smart phone video playback applications are proposed. A Gaussian mixture model (GMM) is used for obtaining the distribution of the real playback length; thereby, the best buffering strategy for minimum energy losses is obtained. In experiments, an Android application is enhanced to monitor and collect the users' YouTube video playback activities. The results show that the proposed strategy ensures remarkable amount of reduction in energy usage when compared to the static strategies. The GMM model outperforms normal and exponential distribution models in terms of energy savings while downloading operations.

For periodic real-time applications, an energy-aware standby-sparing technique is presented in [99]. The use of Earliest Deadline First (EDF), which executes prepared tasks as soon as possible, and Earliest Deadline Late (EDL), which delays the tasks as much as possible, schedules on the primary and collateral CPUs, respectively. The joint use of EDF and EDL allows to minimize the overlap between the two copies at runtime and decrease the energy cost due to the backup implementations. This joint usage forms the Standby-Sparing for Periodic Tasks (SSPT) algorithm. The main idea in the algorithm is that if the primary processor is idle, the job is dispatched immediately. If not, a job is dispatched only if it has a superior priority than the currently executing job. The proposed 
algorithm is compared with reliability-aware power management (RAPM) techniques which assume that the system's original reliability is acceptable when all the tasks are executed at the maximum CPU frequency. The effect of the system load and workload variability is analyzed in simulations. According to results, the SSPT algorithm outperforms RAPM in terms of energy gain.

\section{Conclusions}

Energy conservation has become a very crucial research area to support the enormous growth of the ICT industry. Various analyses indicate that the ICT industry is now responsible for $2 \%$ to $10 \%$ of the global energy consumption and the weight of ICT is expected to increase as we continue to integrate ICT technologies in our daily lives. In this paper, we first review several indicators showing the impact of ICT on energy consumption from data centers to WSNs. Then, we try to describe some recent research studies for all layers of the Internet protocol stack which have addressed the issue of energy efficiency or in other words green communications.

Although the impact of energy consumption for some protocols may be larger than the others, it is very essential to work on all layers to develop less energy-consuming ways for the ICT industry as the increasing levels of energy consumption directly affects the greenhouse gas emissions, global warming, and thus our future.

Most of the energy efficiency studies are observed to be studied in the lower layers. The physical layer is historically the first layer concerned with the energy saving mechanisms and focuses on developing energyefficient modulation schemes. The data link layer concentrates on MAC protocols for energy efficiency. Most of the research in the network layer focuses on developing energy-efficient routing protocols for both wired and wireless networks. Managing the network devices by turning them off (or on) depending on the traffic conditions and data center networks are other extensively studied ways for reducing energy consumption. While in the transport layer new versions of the TCP protocol are studied, the energy efficiency studies for the application layer concentrate on developing power-aware applications and software optimizations.

It is predicted that the growth rate of the ICT industry is going to continue with the invention and widespread usage of new technologies such as IoT (Internet of Things), Cloud Computing, Smart Cities, and Smart Homes. Thus, it is extremely important to develop energyefficient protocols which can satisfy the growing demand with similar or better performance levels both for economical and environmental reasons. Although it might be very difficult, time consuming, and expensive to move from well-established protocols to new ones, it is highly likely that the traditional protocols that we are currently using will be forced to change in the future based on their energy impacts.

\section{Competing interests}

The authors declare that they have no competing interests.

\section{Author details}

${ }^{1}$ Electrical-Electronics Engineering Department, Trakya University, Prof. Dr. Ahmet Karadeniz Yerleskesi, 22030 Edirne, Turkey. ${ }^{2}$ Electrical-Electronics Engineering Department, Kadir Has University, Cibali, 34083 Istanbul, Turkey. ${ }^{3}$ Computer Engineering Department, Kadir Has University, Cibali, 34083 Istanbul, Turkey.

Received: 14 October 2014 Accepted: 20 March 2015

Published online: 18 April 2015

\section{References}

1. G Fettweis, E Zimmermann, in The 11th International Symposium on Wireless Personal Multimedia Communications (WPMC 2008). ICT energy consumption - trends and challenges (Lapland, Finland, 2008)

2. M Pickavet, W Vereecken, S Demeyer, P Audenaert, B Vermeulen, C Develder, D Colle, B Dhoedt, P Demeester, in The 2nd International Symposium on Advanced Networks and Telecommunication Systems (ANTS 2008). Worldwide energy needs for ICT: the rise of power-aware networking (Mumbai, India, 2008), pp. 1-3

3. Committee on America's Climate Choices, Board on Atmospheric Sciences and Climate, Division on Earth and Life Studies, National Research Council, America's Climate Choices. (National Academies Press, 2011)

4. Greenhouse gases, climate change and energy. http://www.eia.gov/oiaf/ 1605/ggccebro/chapter1.html. Accessed 6 April 2015

5. The current and future consequences of global change. http://climate. nasa.gov/effects. Accessed 6 April 2015

6. Effects of global warming. http://environment.nationalgeographic.com/ environment/global-warming/gw-effects/. Accessed 6 April 2015

7. MA Yigitel, OD Incel, C Ersoy, Dynamic base station planning with power adaptation for green wireless cellular networks. EURASIP J. Wireless Commun. Networking. 2014(1), 1-13 (2014)

8. TV Landegem, GreenTouch: Energy consumption in communication networks can be reduced by up to $90 \%$ by the year 2020. http://www. alcatel-lucent.com/blog/corporate/2013/06/greentouch-energyconsumption-communication-networks-can-be-reduced-90-year-2020 Accessed 6 April 2015

9. Bryan Walsh, The surprisingly large energy footprint of the digital economy. http://science.time.com/2013/08/14/power-drain-the-digitalcloud-is-using-more-energy-than-you-think. Accessed 6 April 2015

10. C Marsden, T David-Barrett, E Pavan, in Science.EU Project. Overview of ICT energy consumption (The EINS Consortium, 2013)

11. TRestorick, An inefficient Truth, Global Action Plan Report, (2013)

12. T Asami, S Namiki, in The 34th European Conference and Exhibition on Optical Communication (ECOC 2008). Energy consumption targets for network systems (Brussels, Belgium, 2008), pp. 1-4

13. Energy savings in ICT and ICT for energy savings (2009). http://ercimnews.ercim.eu/en79/special/introduction

14. K Hinton, J Baliga, MZ Feng, RWA Ayre, R Tucker, Power consumption and energy efficiency in the internet. IEEE Network. 25(2), 6-12 (2011)

15. H Sistek, Green-tech base stations cut diesel usage by 80 percent. CNET News Green Tech, ed, (2008)

16. L Suarez, L Nuaymi, J-M Bonnin, An overview and classification of research approaches in green wireless networks. EURASIP J. Wireless Commun. Networking. 2012(1), 1-18 (2012)

17. C Bianco, F Cucchietti, G Griffa, in The 29th International Telecommunications Energy Conference (INTELEC 2007). Energy consumption trends in the next generation access network - a telco perspective (Brussels, Belgium, 2007), pp. 737-742

18. C Keenan, How much energy does the Internet consume? Accessed 6 April 2015. http://planetsave.com/2011/10/27/how-much-energy-doesthe-internet-consume

19. R Bolla, F Davoli, R Bruschi, K Christensen, F Cucchietti, S Singh, The potential impact of green technologies in next-generation wireline 
networks: Is there room for energy saving optimization? Commun. Mag. IEEE. 49(8), 80-86 (2011)

20. C Lange, in 35th European Conference on Optical Communication (ECOC 2009). Energy-related aspects in backbone networks (Wien, Austria, 2009)

21. Stop worrying about IT power consumption. http://www.greenbiz.com/ blog/2013/08/07/jonathan-koomey\-stop-worrying-about-it-powerconsumption. Accessed 6 April 2015

22. D Wei, C Feng, C Guo, L Fangfang, A power amplifier energy efficient polarization modulation scheme based on the optimal pre-compensation. Commun. Lett. IEEE. 17(3), 513-516 (2013). doi:10.1109/LCOMM.2013.012313.122639

23. D Wei, C Feng, C Guo, in Wireless Communications and Networking Conference (WCNC 2013). An energy efficient subcarrier-power allocation scheme for polarization-amplitude-phase modulation in channel with polarization mode dispersion (Shanghai, China, 2013)

24. K Ntontin, M Di Renzo, A Perez-Neira, C Verikoukis, in IEEE International Conference On Communications (ICC 2013). Antenna subset selection for spatial modulation: a novel and energy efficient single RF technique (Budapest, Hungary, June 2013)

25. H Kimura, K Asaka, H Nakamura, S Kimura, N Yoshimoto, in 39th European Conference and Exhibition On Optical Communication (ECOC 2013). First demonstration of energy efficient IM-DD OFDM-PON using dynamic SNR management and adaptive modulation (London, UK, 2013)

26. H Ochiai, in 10th International Symposium On Wireless Communication Systems (ISWCS 2013). On modulation selection for energy-efficient band-limited communication systems (Ilmenau, Germany, 2013)

27. D DiTomaso, S Laha, S Kaya, D Matolak, A Kodi, in 10th IEEE International Conference on New Circuits (NEWCAS 2012). Energy efficient modulation for a wireless network-on-chip architecture (Montreal, Canada, 2012)

28. MS Vasanthi, D Kumar, TR Rao, in International Conference On Advances in Computing, Communications and Informatics (ICACCI 2013). Performance comparison study of AQ-DBPSK modulation scheme for energy efficient wireless sensor communications (Mysore, India, 2013)

29. Z Chen, C Yang, in 8th International Conference On Signal Processing (ICSP 2006). Energy efficiency of cooperative diversity at PHY layer in wireless sensor networks (Beijing, China, 2006)

30. W Feng, JMH Elmirghani, in 3th International Conference On Next Generation Mobile Applications, Services and Technologies. Energy efficiency in ad-hoc wireless networks with two realistic physical layer models (Wales, UK, 2009)

31. A Chehri, HT Mouftah, in 25th IEEE Canadian Conference On Electrical Computer Engineering (CCECE 2012). Qos aware green routing protocol for wireless sensor networks (Montreal, Canada, 2012)

32. D Dessales, A Poussard, R Vauzelle, N Richard, F Gaudaire, C Martinsons, in Conference On Design and Architectures for Signal and Image Processing (DASIP 2010). Physical layer study in a goal of robustness and energy efficiency for wireless sensor networks (Edinburg, Scotland, 2010)

33. A Bartoli, J Hernandez-Serrano, M Soriano, M Dohler, A Kountouris, D Barthel, in IEEE Globecom Workshops (GC Wkshps 2012). Optimizing energy-efficiency of PHY-layer authentication in machine-to-machine networks (Anaheim, CA, 2012)

34. R Zhang, C Comaniciu, HV Poor, in 16th International Symposium On Wireless Personal Multimedia Communications (WPMC 2013). Outage capacity and partial secrecy for energy efficient physical layer security in gaussian fading channels (NJ, USA, 24)

35. X Chen, L Lei, Energy-efficient optimization for physical layer security in multi-antenna downlink networks with QoS guarantee. Commun. Lett. IEEE. 17(4), 637-640 (2013)

36. I Mobin, RJ Mondragon, MM Khan, in 4th Conference on Computer Science and Electronic Engineering (CEEC 2012). Energy efficient transmission power estimation for WLAN VoIP (Colchester, UK, 2012)

37. KR Malekshan, W Zhuang, in IEEE International Conference On Communications (ICC 2013). An energy efficient MAC protocol for fully-connected wireless networks (Budapest, Hungary, 2013)

38. N Abdeddaim, F Theoleyre, M Heusse, A Duda, in IEEE International Conference On Distributed Computing in Sensor Systems (DCOSS 2013). Adaptive IEEE 802.15.4 MAC for throughput and energy optimization (Massachusetts, USA, 2013)

39. SToklu, OA Erdem, BSC-MAC: energy efficiency in wireless sensor networks with base station control. Comput. Networks. 59(0), 91-100 (2014)
40. A Nasir, B-H Soong, in 8th International Conference On Information, Communications and Signal Processing (ICICS 2011). Energysim; a novel, fast, extensible wireless sensor network mac protocol simulator for evaluating energy efficiency (Singapore, 2011)

41. MR Sabagh, M Dianati, MA Imran, R Tafazolli, in IEEE International Conference On Communications (ICC 2013). A heuristic energy efficient scheduling scheme for voip in 3GPP LTE networks (Budapest, Hungary, 2013)

42. J-M Liang, J-J Chen, H-H Cheng, Y-C Tseng, in 9th International Conference on Wireless Communications and Mobile Computing (IWCMC 2013). Energy-efficient sleep scheduling with QoS considerations in 3GPP LTE-advanced networks (Cagliari, Italy, 2013)

43. FD Cardoso, S Petersson, M Boldi, S Mizuta, G Dietl, R Torrea-Duran, C Desset, J Leinonen, LM Correia, Energy efficient transmission techniques for LTE. Commun. Mag. IEEE. 51(10), 182-190 (2013)

44. M Deruyck, W Joseph, B Lannoo, D Colle, L Martens, Designing energy-efficient wireless access networks: LTE and LTE-advanced. IEEE Internet Comput. 17(5), 39-45 (2013)

45. A Cianfrani, V Eramo, M Listanti, M Marazza, E Vittorini, in IEEE Conference on Computer Communications Workshops (INFOCOM 2010). An energy saving routing algorithm for a green OSPF protocol (San Diego, USA, 2010)

46. SSW Lee, P-K Tseng, A Chen, in IEEE Global Telecommunications Conference (GLOBECOM 2011). A distributed link management algorithm for energy efficient IP networks (Texas, USA, 2011)

47. AP Bianzino, L Chiaraviglio, M Mellia, in IEEE Conference On Computer Communications Workshops (INFOCOM 2012). Distributed algorithms for green IP networks (Florida, USA, 2012)

48. P Lai, Q Yang, C Wu, M Jiang, in IEEE 3rd International Conference On Communication Software and Networks (ICCSN 2011). Configuring network topology towards energy-efficient IP networks (Xi'an, China, 2011)

49. A Coiro, M Polverini, A Cianfrani, M Listanti, in International Conference On Computing, Networking and Communications (ICNC 2013). Energy saving improvements in IP networks through table lookup bypass in router line cards (San Diego, USA, 2013)

50. Q Li, M Xu, Y Yang, L Gao, Y Cui, J Wu, Safe and Practical Energy-Efficient Detour Routing in IP Networks. IEEE Trans. Networking. 22(6), 1925-1937 (2013)

51. F Francois, N Wang, K Moessner, S Georgoulas, KXu, in IFIP Networking Conference (2013). Green IGP link weights for energy-efficiency and load-balancing in IP backbone networks (New York, USA, 2013)

52. A Coiro, M Listanti, A Valenti, F Matera, Energy-aware traffic engineering: a routing-based distributed solution for connection-oriented IP networks. Comput. Networks. 57(9), 2004-2020 (2013)

53. E Amaldi, A Capone, LG Gianoli, Energy-aware IP traffic engineering with shortest path routing. Comput. Networks. 57(6), 1503-1517 (2013)

54. AP Bianzino, C Chaudet, F Larroca, D Rossi, J Rougier, in IEEE Global Telecommunications Conference(GLOBECOM 2011). Energy-aware routing: a reality check (Texas, USA, 2011)

55. M Fiorani, M Casoni, S Aleksic, Hybrid optical switching for an energy-efficient Internet core. IEEE Internet Comput. 17(1), 14-22 (2013)

56. A Klekamp, U Gebhard, F Ilchmann, Energy and cost efficiency of adaptive and mixed-line-rate IP over DWDM networks. J Lightwave Technol. 30(2), 215-221 (2012)

57. X Dong, TEH El-Gorashi, JMH Elmirghani, On the energy efficiency of physical topology design for IP over WDM networks. J Lightwave Technol. 30(12), 1931-1942 (2012)

58. K Ramanujam, S Talbatulla, in International Conference On Optical Engineering (ICOE 2012). Greening the IP over optical backbone network (Belgaum, India, 2012)

59. X Dong, TEH El-Gorashi, JMH Elmirghani, in IEEE International Conference On Communications (ICC 2013). Energy efficiency of optical OFDM-based networks (Budapest, Hungary, 2013)

60. Data Center power consumption continuing to grow with Cloud Computing as an option. Accessed 6 April 2015. http://research.gigaom com/2013/02/data-center-power-consumption-continuing-to-growwith-cloud-computing-as-an-option

61. Federal Energy Management Program. Accessed 6 April 2015. http:// www.energy.gov/node/782456/program

62. Google Green. Accessed 6 April 2015. http://www.google.com/green/ bigpicture 
63. R Barga, in IEEE 9th International Conference On Peer-to-Peer Computing (P2P 2009). Cloud computing-a microsoft research perspective (Washington, USA, 2009)

64. X Dong, T El-Gorashi, JMH Elmirghani, in 13th International Conference On Transparent Optical Networks (ICTON 2011). Energy-efficient IP over WDM networks with data centres (Stockholm, Sweden, 2011)

65. M Zapater, JL Ayala, JM Moya, K Vaidyanathan, K Gross, AK Coskun, in Design, Automation Test in Europe Conference Exhibition (DATE 2013). Leakage and temperature aware server control for improving energy efficiency in data centers (Grenoble, France, 2013)

66. J Qu, L Li, L Liu, Y Tian, J Chen, in IEEE International Conference On Service Operations and Logistics, and Informatics (SOLI 2013). Smart temperature monitoring for data center energy efficiency (Dongguan, China, 2013)

67. NM Asghari, M Mandjes, A Walid, Energy-efficient scheduling in multi-core servers. Comput. Networks. 59(0), 33-43 (2014)

68. M Xu, Y Shang, D Li, X Wang, Greening data center networks with throughput-guaranteed power-aware routing. Comput. Networks. 57(15), 2880-2899 (2013)

69. Y Shang, D Li, M Xu, in 19th IEEE Workshop On Local Metropolitan Area Networks (LANMAN 2013). Greening data center networks with flow preemption and energy-aware routing (Brussels, Belgium, 2013)

70. BS Gouda, AK Dass, KL Narayana, in International Multi-Conference On Automation, Computing, Communication, Control and Compressed Sensing (iMac4s 2013). A comprehensive performance analysis of energy efficient routing protocols in different traffic based mobile ad-hoc networks (Kerala, India, 2013)

71. T Zhu, D Towsley, in IEEE Conference On Computer Communications Workshops (INFOCOM 2011). E2r: Energy efficient routing for multi-hop green wireless networks (Shanghai, China, 2011)

72. J Zhu, X Wang, Model and protocol for energy-efficient routing over mobile ad hoc networks. IEEE Trans. Mobile Comput. 10(11), 1546-1557 (2011)

73. S Zarifzadeh, N Yazdani, A Nayyeri, Energy-efficient topology control in wireless ad hoc networks with selfish nodes. Comput. Networks. 56(2), 902-914 (2012)

74. C Lozano-Garzon, M Camelo, P Vila, Y Donoso, in International Symposium On Performance Evaluation of Computer and Telecommunication Systems (SPECTS 2013). Green routing algorithm for wireless mesh network: a multi-objective evolutionary approach (Toronto, Canada, 2013)

75. Y Zeng, J Wu, N Xiong, D Li, in 32nd International Conference On Distributed Computing Systems Workshops (ICDCSW 2012). Energy-efficient routing and rate allocation for delay tolerant networks (Macau, China, 2012)

76. WR Heinzelman, A Chandrakasan, H Balakrishnan, in 33rd Annual Hawaii International Conference On System Sciences. Energy-efficient communication protocol for wireless microsensor networks (Maui, Hawaii, 2000)

77. MO Farooq, AB Dogar, GA Shah, in 4th International Conference On Sensor Technologies and Applications (SENSORCOMM 2010). MR-LEACH: multi-hop routing with low energy adaptive clustering hierarchy (Venice, Italy, 2010)

78. N Kumar, J Kaur, in 7th International Conference On Wireless Communications, Networking and Mobile Computing (WiCOM 2011). Improved leach protocol for wireless sensor networks (Wuhan, China, 2011)

79. X Hu, J Luo, Z Xia, M Hu, in IEEE 3rd International Conference On Communication Software and Networks (ICCSN 2011). Adaptive algorithm of cluster head in wireless sensor network based on LEACH (Xi'an, China, 2011)

80. N Kumar, P Bhutani, P Mishra, et al., in International Conference On Communication, Information \& Computing Technology (ICCICT 2012). U-LEACH: a novel routing protocol for heterogeneous wireless sensor networks (Mumbai, India, 2012)

81. R Mehta, A Pandey, P Kapadia, in International Conference On Computer Communication and Informatics (ICCCI 2012). Reforming clusters using C-LEACH in wireless sensor networks (Coimbatore, India, 2012)

82. H-W Ferng, D Rachmarini, in IEEE Network Operations and Management Symposium (NOMS 2012). A secure routing protocol for wireless sensor networks with consideration of energy efficiency (Hawaii, USA, 2012)

83. M Tembhurkar, L Malik, N Chavhan, in 8th International Conference On Computer Science Education (ICCSE 2013). Energy efficient VoIP communication using WSN clustering approach (Colombo, Sri Lanka, 2013)
84. NA Pantazis, SA Nikolidakis, DD Vergados, Energy-efficient routing protocols in wireless sensor networks: a survey. IEEE Commun. Surveys Tutorials. 15(2), 551-591 (2013)

85. Y Fukuda, S Kawamata, T Ikenaga, Y Oie, in IEEE International Conference On Green Computing and Communications (GreenCom 2012). Performance evaluation of the transmission control protocols with the dynamic link control scheme for power saving (Besançon, France, 2012)

86. TA Le, C-S Hong, MA Razzaque, S Lee, H Jung, ecMTCP: an energy-aware congestion control algorithm for multipath TCP. IEEE Commun. Lett. 16(2), 275-277 (2012)

87. S Chen, Z Yuan, G-M Muntean, in IEEE Wireless Communications and Networking Conference (WCNC 2013). An energy-aware multipath-TCP-based content delivery scheme in heterogeneous wireless networks (Shanghai, China, 2013)

88. A Sassu, C Scarso, F Cuomo, in The 2nd IFIP Conference on Sustainable Internet and ICT for Sustainability (Sustain/T 2012). TCP behavior over a greened network (Pisa, Italy, 2012)

89. P Ramya, K Radha, Design of efficient transport protocol for multimedia through wireless networks. Int. J. Recent Technol. Eng. (IJRTE). 02(03), 1-4 (2013)

90. M Oulmahdi, C Chassot, E Exposito, in International Conference On Smart Communications in Network Technologies (SaCoNeT 2013). An energy-aware TCP for multimedia streaming (Paris, France, 2013)

91. KK Sharma, RB Patel, H Singh, A reliable and energy efficient transport protocol for wireless sensor networks. Int. J. Comput. Networks Commun. (IJCNC). 02(05), 1-12 (2010)

92. G Han, J Lu, X Gan, H Li, W Dou, DREET: a data-reliable, energy-efficient transport layer protocol. J. Software. 07(09), 1925-1931 (2012)

93. K Sasikala, R Wahidabanu, Rate based transport protocol using delay information for reducing energy consumption of nodes in manet. Int. J. Eng. Technol. 05(05), 1-10 (2012)

94. P Bartalos, MB Blake, S Remy, in IEEE International Conference On ServiceOriented Computing and Applications (SOCA 2011). Green web services: models for energy-aware web services and applications (Irvine, USA, 2011)

95. BK Donohoo, C Ohlsen, S Pasricha, in IEEE 29th International Conference On Computer Design (ICCD 2011). AURA: an application and user interaction aware middleware framework for energy optimization in mobile devices (Amherst, USA, 2011)

96. A Shye, B Scholbrock, G Memik, in 42nd Annual IEEE/ACM International Symposium On Microarchitecture (MICRO-42 2009). Into the wild: studying real user activity patterns to guide power optimizations for mobile architectures (New York, USA, 2009)

97. H Chen, B Luo, W Shi, in 41st International Conference On Parallel Processing Workshops (ICPPW 2012). Anole: A case for energy-aware mobile application design (Pittsburgh, PA, 2012)

98. H Shen, Q Qiu, in Design, Automation Test in Europe Conference Exhibition (DATE 2013). User-aware energy efficient streaming strategy for smartphone based video playback applications (Grenoble, France, 2013)

99. MA Haque, H Aydin, D Zhu, in IEEE 29th International Conference On Computer Design (ICCD 2011). Energy-aware standby-sparing technique for periodic real-time applications (Amherst, USA, 2011)

\section{Submit your manuscript to a SpringerOpen ${ }^{\circ}$ journal and benefit from:}

- Convenient online submission

Rigorous peer review

- Immediate publication on acceptance

- Open access: articles freely available online

- High visibility within the field

- Retaining the copyright to your article

Submit your next manuscript at $>$ springeropen.com 